\title{
Flow in pipes with non-uniform curvature and torsion
}

\author{
By DAVID GAMMACK AND PETER E. HYDON \\ Dept. of Mathematics and Statistics, University of Surrey, Guildford, GU2 7XH, UK
}

(Received 17 July 1997 and in revised form 21 August 2000)

This paper describes steady and unsteady flows in pipes with small, slowly varying curvature and torsion. Four new pipe shapes are studied, using Germano's extension of the Dean equations. Analytic and numerical solutions are obtained for flows driven by a steady pressure gradient. Oscillatory flows in pipes with non-uniform curvature are obtained by numerical methods. The effects of the non-uniformities in curvature and torsion are discussed, with particular reference to wall shear stress.

\section{Introduction}

Hardening of the arteries (atherosclerosis) is the main cause of cardiac failure. There is still some debate on the causes of atherosclerosis, but there is evidence that it is initiated in regions where the arterial wall shear stress is low (Caro, Fitz-Gerald $\&$ Schroter 1971). Therefore the study of flow in curved and twisted pipes contributes to the understanding of this phenomenon.

Dean $(1927,1928)$ analysed steady flow in a loosely coiled pipe and showed that, when the Dean number is sufficiently small, the secondary flow consists of a pair of counter-rotating vortices. Numerical studies of toroidal pipes have shown that, under certain conditions, a four-vortex solution exists for both steady flow (Dennis \& Ng 1982; Nandakumar \& Masliyah 1982; Yanase, Gotoh \& Yamamoto 1989) and unsteady flow (Lyne 1971; Zalosh \& Nelson 1973). A stable four-vortex solution was also observed in the experimental work of Bertelsen (1975).

Until now, analytical studies of steady flow in helical pipes (Germano 1982, 1989; Kao 1987; Tuttle 1990; Wang 1981) have focused on uniform geometries. Tuttle (1990) and Kao (1987) showed that, for pipes with circular cross-section, torsion causes a skewing of Dean's two-vortex structure. The numerical work by Liu \& Masliyah (1993) showed that a single-vortex solution exists provided that the torsion is much greater than the curvature. Yamamoto, Yanase \& Yoshida (1994) used numerical techniques to solve the full Navier-Stokes equations for a wide range of parameters; they found no evidence that helical pipes admit a stable four-vortex solution. Hüttl \& Friedrich $(1999 a, b)$ and Hüttl, Wagner \& Friedrich (1999) used Germano's coordinate system to compute laminar and turbulent flows in curved and helical pipes. Germano's coordinates have two main advantages: they are suitable for a very wide class of pipe shapes, and they are orthogonal. However, they also have a significant disadvantage for a helical pipe: they are referred to the pipe centreline, and so Germano's basis does not contain the tangent vector field to the line congruence of helices with the same fixed pitch. (The line congruence can be visualized as a set of helical hairs filling the pipe; it provides a natural means of distinguishing between the axial and secondary components of the flow.) Zabielski \& Mestel $(1998 a, b)$ discussed the above difficulty, and 
used a helically symmetric coordinate system to calculate steady and unsteady flows. They found evidence of symmetry breaking in the unsteady case. Zabielski \& Mestel also showed that at large Reynolds numbers the solution has an asymptotic structure that is qualitatively similar to the curved pipe solution of Hüttl \& Friedrich (1999a).

The human arterial system is non-uniform. Some arteries curve and bifurcate out of plane. For example, the aortic arch and the bifurcation of the aorta have non-planar geometry (Caro et al. 1996). Arteries have distensible walls and non-uniform crosssections; they may also move within the body. Therefore the modelling of arterial flow is a substantial challenge. Some progress has been made by considering each of the main features of arterial flow separately. Murata, Miyake \& Inaba (1976) studied steady flow in pipes with zero torsion and non-uniform curvature, and found that the Reynolds number affects the position of the maximum axial velocity. Other features that have been studied include arterial movement (Lynch, Waters \& Pedley 1996; Schilt et al. 1996; Waters 1996), taper (Grotberg 1984), bifurcation, and elasticity (see the review by Pedley 1995).

The current paper is an analysis of flow in non-uniformly curved and twisted pipes. Various coordinate systems have been used to describe flows in pipes with uniform torsion. Germano $(1982,1989)$ used an orthogonal coordinate system that rotates with torsion, whereas Wang (1981) and Liu \& Masliyah (1993) used a nonorthogonal system that allows the definition of a secondary flow (pseudo-) stream function. Kao (1987) demonstrated that no stream function exists in the orthogonal system, and so the secondary flow appears to contain distributed sources and sinks. Tuttle (1990) gives an excellent review of both coordinate systems, showing that the results of Wang and Germano are consistent with each other. Zabielski \& Mestel (1999a) argue that, for helical pipes, a helically symmetric coordinate system is the most appropriate. However, the aim of this paper is to gain some insight into the effects of non-uniform curvature and torsion; we consider pipes that lack helical symmetry. In particular, we want to find an analytic description of the flow, which can be achieved by using Germano's orthogonal system.

Germano begins with the Navier-Stokes equations for general curvature, $\hat{\kappa}$, and torsion, $\hat{\tau}$, and then expands in powers of the dimensionless curvature parameter. This allows the Dean equations to be extended to uniform helical-pipe flow. We use Germano's equations for general helical flow and solve for non-uniformly curved and twisted pipes by allowing $\hat{\kappa}$ and $\hat{\tau}$ to vary exponentially with the arc-length, $\hat{s}$. This yields a variety of pipe shapes, including the uniform helical pipe (enabling some of our results to be compared with those of Kao, Liu \& Masliyah, and Tuttle). Our analytical solution is valid for pipes with slowly varying curvature and torsion. We also present numerical solutions for various cases in which the analytical solution breaks down.

\section{Governing equations}

Germano (1982, 1989) constructed a rotating coordinate system (figure 1) by considering a spatial curve described by the position vector $\hat{\boldsymbol{R}}(\hat{s})$. (A caret over a variable indicates that it is a dimensional quantity.) The curve defines the orthonormal triad $\boldsymbol{T}, \boldsymbol{N}, \boldsymbol{B}$, which are respectively the tangent, normal, and binormal vectors to the centreline. Germano demonstrated that, using this system, any Cartesian vector $\hat{\boldsymbol{x}}$ can be expressed as

$$
\hat{\boldsymbol{x}}=\hat{\boldsymbol{R}}(\hat{s})+\hat{r} \cos \left(\theta+\phi(\hat{s})+\phi_{0}\right) \boldsymbol{N}(\hat{s})+\hat{r} \sin \left(\theta+\phi(\hat{s})+\phi_{0}\right) \boldsymbol{B}(\hat{s}) .
$$




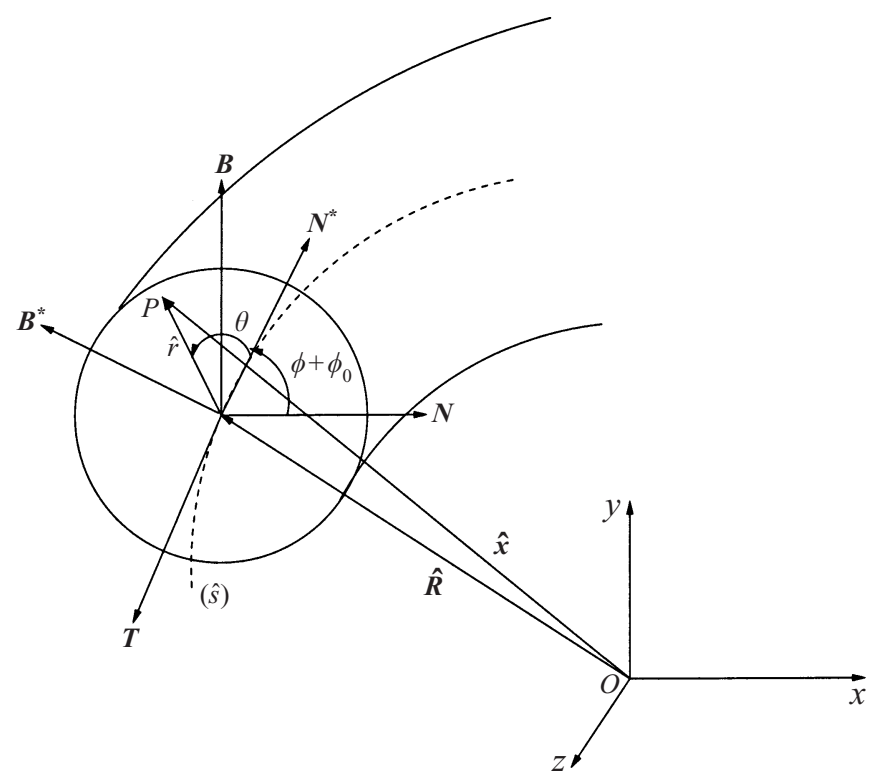

FIGURE 1. The coordinate system.

Here $(\hat{r}, \theta)$ are polar coordinates on the cross-section. The azimuthal angle, $\theta$, is measured from the unit vector $N^{*}$ which is rotated from $N$ by $\phi+\phi_{0}$, where

$$
\phi(\hat{s})=-\int^{\hat{s}} \hat{\tau}\left(s^{\prime}\right) \mathrm{d} s^{\prime},
$$

and $\phi_{0}$ is an arbitrary constant angle. Following Germano, we set $\phi_{0}=\pi / 2$. Using the relations

and the Serret-Frenet formulae

$$
\boldsymbol{T}=\frac{\mathrm{d} \hat{\boldsymbol{R}}}{\mathrm{d} \hat{s}}, \quad \boldsymbol{B}=\boldsymbol{T} \times \boldsymbol{N}
$$

$$
\frac{\mathrm{d} \boldsymbol{T}}{\mathrm{d} \hat{s}}=\hat{\kappa} \boldsymbol{N}, \quad \frac{\mathrm{d} \boldsymbol{N}}{\mathrm{d} \hat{s}}=\hat{\tau} \boldsymbol{B}-\hat{\kappa} \boldsymbol{T}, \quad \frac{\mathrm{d} \boldsymbol{B}}{\mathrm{d} \hat{s}}=-\hat{\tau} \boldsymbol{N},
$$

Germano derived the metric

$$
\mathrm{d} \hat{\boldsymbol{x}} \cdot \mathrm{d} \hat{\boldsymbol{x}}=[1+\hat{\kappa} \hat{r} \sin (\theta+\phi(\hat{s}))]^{2}(\mathrm{~d} \hat{s})^{2}+(\mathrm{d} \hat{r})^{2}+\hat{r}^{2}(\mathrm{~d} \theta)^{2} .
$$

Therefore the Navier-Stokes equations are

$$
\begin{gathered}
\varpi \frac{\partial \hat{u}}{\partial \hat{s}}+\frac{\partial \hat{v}}{\partial \hat{r}}+\frac{1}{\hat{r}} \frac{\partial \hat{w}}{\partial \theta}+\frac{\hat{v}}{\hat{r}}+\hat{\kappa} \varpi[\hat{v} \sin (\theta+\phi)+\hat{w} \cos (\theta+\phi)]=0, \\
\frac{\partial \hat{u}}{\partial \hat{t}}+\hat{\mathscr{D}} \hat{u}+\hat{\kappa} \varpi \hat{u}[\hat{v} \sin (\theta+\phi)+\hat{w} \cos (\theta+\phi)] \\
=-\varpi \frac{1}{\rho_{0}} \frac{\partial \hat{p}}{\partial \hat{s}}+v\left[\left(\frac{\partial}{\partial \hat{r}}+\frac{1}{\hat{r}}\right)\left(\frac{\partial \hat{u}}{\partial \hat{r}}+\hat{\kappa} \varpi \hat{u} \sin (\theta+\phi)-\varpi \frac{\partial \hat{v}}{\partial \hat{s}}\right)\right. \\
\left.+\frac{1}{\hat{r}} \frac{\partial}{\partial \theta}\left(\frac{1}{\hat{r}} \frac{\partial \hat{u}}{\partial \theta}+\hat{\kappa} \varpi \hat{u} \cos (\theta+\phi)-\varpi \frac{\partial \hat{w}}{\partial \hat{s}}\right)\right],
\end{gathered}
$$




$$
\begin{aligned}
\frac{\partial \hat{v}}{\partial \hat{t}}+\hat{\mathscr{D}} \hat{v}- & \frac{\hat{w}^{2}}{\hat{r}}-\hat{\kappa} \varpi \hat{u}^{2} \sin (\theta+\phi) \\
= & -\frac{1}{\rho_{0}} \frac{\partial \hat{p}}{\partial \hat{r}}-v\left[\left(\frac{1}{\hat{r}} \frac{\partial}{\partial \theta}+\hat{\kappa} \varpi \cos (\theta+\phi)\right)\left(\frac{\partial \hat{w}}{\partial \hat{r}}+\frac{\hat{w}}{\hat{r}}-\frac{1}{\hat{r}} \frac{\partial \hat{v}}{\partial \theta}\right)\right. \\
& \left.-\varpi \frac{\partial}{\partial \hat{s}}\left(\varpi \frac{\partial \hat{v}}{\partial \hat{s}}-\frac{\partial \hat{u}}{\partial \hat{r}}-\hat{\kappa} \varpi \hat{u} \sin (\theta+\phi)\right)\right],
\end{aligned}
$$

$$
\begin{aligned}
\frac{\partial \hat{w}}{\partial \hat{t}}+\hat{\mathscr{D}} \hat{w}+ & \frac{\hat{v} \hat{w}}{\hat{r}}-\hat{\kappa} \varpi \hat{u}^{2} \cos (\theta+\phi) \\
= & -\frac{1}{\hat{r} \rho_{0}} \frac{\partial \hat{p}}{\partial \theta}+v\left[\left(\frac{\partial}{\partial \hat{r}}+\hat{\kappa} \varpi \sin (\theta+\phi)\right)\left(\frac{\partial \hat{w}}{\partial \hat{r}}+\frac{\hat{w}}{\hat{r}}-\frac{1}{\hat{r}} \frac{\partial \hat{v}}{\partial \theta}\right)\right. \\
& \left.-\varpi \frac{\partial}{\partial \hat{s}}\left(\frac{1}{\hat{r}} \frac{\partial \hat{u}}{\partial \theta}+\hat{\kappa} \varpi \hat{u} \cos (\theta+\phi)-\varpi \frac{\partial \hat{w}}{\partial \hat{s}}\right)\right],
\end{aligned}
$$

where

$$
\varpi=\frac{1}{1+\hat{\kappa} \hat{r} \sin (\theta+\phi)}, \quad \hat{\mathscr{D}}=\varpi \hat{u} \frac{\partial}{\partial \hat{s}}+\hat{v} \frac{\partial}{\partial \hat{r}}+\frac{\hat{w}}{\hat{r}} \frac{\partial}{\partial \theta} .
$$

Here $(\hat{u}, \hat{v}, \hat{w})$ are the axial, radial and azimuthal components of the velocity field, $\hat{p}$ is the pressure, $v$ is the kinematic viscosity, and $\rho_{0}$ is the (uniform) fluid density. The boundary conditions are the no-slip condition, i.e. $(u, v, w)=(0,0,0)$ at the pipe wall, and that the velocities and stresses are finite throughout the cross-section.

\section{Steady flow}

Following Germano, we express the Navier-Stokes equations in terms of the nondimensionalized variables

$$
(u, v, w)=\left(\frac{\hat{u}}{U_{0}}, \frac{\hat{v}}{U_{0}}, \frac{\hat{w}}{U_{0}}\right), \quad p=\frac{\hat{p}}{\rho_{0} U_{0}^{2}}, \quad s=\frac{\hat{s}}{a}, \quad r=\frac{\hat{r}}{a}, \quad \phi_{0}=\frac{\pi}{2},
$$

where $U_{0}$ is the cross-sectionally averaged velocity in the limit as the curvature tends to zero and $a$ is the pipe radius. The dimensionless parameters are

$$
R e=\frac{U_{0} a}{v}, \quad \lambda(s)=\frac{\hat{\tau}(\hat{s})}{\hat{\kappa}(\hat{s})}, \quad \epsilon(s)=\hat{\kappa}(\hat{s}) a .
$$

Here $R e$ is the Reynolds number, $\epsilon$ is the local curvature parameter and $\lambda$ is the local ratio of torsion to curvature. The coordinates $(s, r, \xi)$ are used, where $\xi=\theta+\phi(\hat{s})$. In this coordinate system, the Navier-Stokes equations for incompressible steady flow are

$$
\begin{gathered}
\varpi\left(u_{s}-\epsilon \lambda u_{\xi}\right)+v_{r}+\frac{w_{\xi}}{r}+\frac{v}{r}+\epsilon \varpi[v \sin \xi+w \cos \xi]=0, \\
\varpi u u_{s}+v u_{r}+\left(\frac{w}{r}-\epsilon \lambda \varpi u\right) u_{\xi}+\epsilon \varpi u[v \sin \xi+w \cos \xi] \\
=-\varpi p_{s}+\epsilon \lambda \varpi p_{\xi}+\frac{1}{R e}\left[\left(\frac{\partial}{\partial r}+\frac{1}{r}\right)\left(u_{r}+\epsilon \varpi u \sin \xi-\varpi v_{s}+\epsilon \lambda \varpi v_{\xi}\right)\right. \\
\left.+\frac{1}{r} \frac{\partial}{\partial \xi}\left(\frac{u_{\xi}}{r}-\varpi w_{s}+\epsilon \varpi u \cos \xi+\epsilon \lambda \varpi w_{\xi}\right)\right]
\end{gathered}
$$




$$
\begin{aligned}
& \varpi u v_{s}+v v_{r}+\left(\frac{w}{r}-\epsilon \lambda \varpi u\right) v_{\xi}-\frac{w^{2}}{r}-\epsilon \varpi u^{2} \sin \xi \\
&=-p_{r}+\frac{1}{R e}\left[\left(\frac{1}{r} \frac{\partial}{\partial \xi}+\epsilon \varpi \cos \xi\right)\left(-w_{r}-\frac{w}{r}+\frac{v_{\xi}}{r}\right)\right. \\
&\left.+\varpi\left(\frac{\partial}{\partial s}-\epsilon \lambda \frac{\partial}{\partial \xi}\right)\left(\varpi v_{s}-u_{r}-\epsilon \varpi u \sin \xi-\epsilon \lambda \varpi v_{\xi}\right)\right] \\
& \varpi u w_{s}+v w_{r}+ \frac{\left(\frac{w}{r}-\epsilon \lambda \varpi u\right) w_{\xi}+\frac{v w}{r}-\epsilon \varpi u^{2} \cos \xi}{=-} \\
&-\frac{p_{\xi}}{r}+\frac{1}{R e}\left[\left(\frac{\partial}{\partial r}+\epsilon \varpi \sin \xi\right)\left(w_{r}+\frac{w}{r}-\frac{v_{\xi}}{r}\right)\right. \\
&\left.-\varpi\left(\frac{\partial}{\partial s}-\epsilon \lambda \frac{\partial}{\partial \xi}\right)\left(-\varpi w_{s}+\frac{u_{\xi}}{r}+\epsilon \varpi u \cos \xi+\epsilon \lambda \varpi w_{\xi}\right)\right]
\end{aligned}
$$

where

$$
\varpi=\frac{1}{1+\epsilon r \sin \xi}=1-\epsilon r \sin \xi+\epsilon^{2} r^{2} \sin ^{2} \xi+O\left(\epsilon^{3}\right) .
$$

Unlike Germano, we cannot set the $s$-derivatives to zero, because $\epsilon$ and $\lambda$ depend on $s$.

\subsection{The expansion}

To study the effects of non-uniform curvature and torsion we assume that the curvature and torsion parameters $(\epsilon, \epsilon \lambda)$ are small in magnitude over a range of $s$. This allows us to follow the work of Dean and perturb about Poiseuille flow. Therefore we look for solutions of (3.1) of the form:

$$
\begin{aligned}
u & =u_{0}(r)+\epsilon(s) u_{1}(s, r) \sin (\xi)+\epsilon(s)^{2} u_{2}(s, r, \xi)+O\left(\epsilon(s)^{3}\right), \\
v & =\epsilon(s) v_{1}(s, r) \sin (\xi)+\epsilon(s)^{2} v_{2}(s, r, \xi)+O\left(\epsilon(s)^{3}\right), \\
w & =\epsilon(s) w_{1}(s, r) \cos (\xi)+\epsilon(s)^{2} w_{2}(s, r, \xi)+O\left(\epsilon(s)^{3}\right), \\
p & =p_{0}(s)+\epsilon(s) p_{1}(s, r) \sin (\xi)+\epsilon(s)^{2} p_{2}(s, r, \xi)+O\left(\epsilon(s)^{3}\right) .
\end{aligned}
$$

The leading-order terms describe Poiseuille flow:

$$
u_{0}=2\left(1-r^{2}\right), \quad p_{0}=-\frac{8 s}{R e} .
$$

The $O(\epsilon)$-equations are as follows:

$$
\begin{gathered}
u_{1, s}+\frac{\dot{\epsilon}}{\epsilon} u_{1}+v_{1, r}-\frac{w_{1}}{r}+\frac{v_{1}}{r}=0 \\
u_{0} u_{1, s}+\frac{\dot{\epsilon}}{\epsilon} u_{0} u_{1}+u_{0}^{\prime} v_{1}=-p_{1, s}-\frac{\dot{\epsilon}}{\epsilon} p_{1}-\frac{8 r}{R e}+\frac{1}{R e}\left[\left(\frac{\partial}{\partial r}+\frac{1}{r}\right)\left(u_{1, r}+u_{0}-\frac{\dot{\epsilon}}{\epsilon} v_{1}-v_{1, s}\right)\right. \\
\left.\quad-\frac{u_{1}}{r^{2}}-\frac{u_{0}}{r}+\frac{\dot{\epsilon}}{\epsilon} \frac{w_{1}}{r}+\frac{w_{1, s}}{r}\right] \\
u_{0} v_{1, s}+\frac{\dot{\epsilon}}{\epsilon} u_{0} v_{1}-u_{0}^{2}=-p_{1, r}+\frac{1}{R e}\left[\frac{1}{\epsilon} \frac{\partial}{\partial s}\left(\epsilon\left(-u_{1, r}-u_{0}+\frac{\dot{\epsilon}}{\epsilon} v_{1}+v_{1, s}\right)\right)\right. \\
\left.+\frac{w_{1, r}}{r}+\frac{w_{1}}{r^{2}}-\frac{v_{1}}{r^{2}}\right]
\end{gathered}
$$




$$
\begin{array}{r}
u_{0} w_{1, s}+\frac{\dot{\epsilon}}{\epsilon} u_{0} w_{1}-u_{0}^{2}=-\frac{p_{1}}{r}+\frac{1}{\operatorname{Re}}\left[\frac{1}{\epsilon} \frac{\partial}{\partial s}(\epsilon(-\right. \\
\left.\left.\frac{u_{1}}{r}-u_{0}+\frac{\dot{\epsilon}}{\epsilon} w_{1}+w_{1, s}\right)\right), \\
\left.+\frac{\partial}{\partial r}\left(w_{1, r}+\frac{w_{1}}{r}-\frac{v_{1}}{r}\right)\right],
\end{array}
$$

where a dot represents the derivative with respect to $s$. We now restrict attention to pipes whose curvature varies exponentially, i.e. $\epsilon=\kappa_{0} \mathrm{e}^{\eta s}$ for some constants $\eta$ and $\kappa_{0}$. The advantage of doing this is that (3.5)-(3.8) has solutions that are independent of $s$. Moreover, if $\epsilon$ is an arbitrary smooth function of $s$, we may approximate it locally as an exponential by letting

$$
\eta=\left.\frac{\mathrm{d} \ln \epsilon}{\mathrm{d} s}\right|_{s=0} .
$$

By Taylor's theorem, the error is $O\left((\eta s)^{2}\right)$ which is acceptably small if $\eta s \leqslant 1$. Using the substitutions

$$
u_{1}=r f(\rho), \quad v_{1}=g(\rho), \quad w_{1}=h(\rho), \quad p_{1}=r q(\rho), \quad \text { where } \rho=r^{2},
$$

we obtain

$$
\left.\begin{array}{rl}
\eta f+2 g^{\prime}-\frac{h}{\rho}+\frac{g}{\rho} & =0, \\
\eta u_{0} f+\frac{u_{0}^{\prime}}{r} g & =-\eta q-\frac{8}{R e}+\frac{1}{R e}\left[4 \rho f^{\prime \prime}+8 f^{\prime}-2 \eta g^{\prime}-\frac{\eta}{\rho} g+\frac{\eta}{\rho} h+\frac{u_{0}^{\prime}}{r}\right], \\
\eta u_{0} g-u_{0}^{2} & =-2 \rho q^{\prime}-q+\frac{1}{R e}\left[-2 \eta \rho f^{\prime}-\eta f-\frac{g}{\rho}+\eta^{2} g+2 h^{\prime}+\frac{h}{\rho}-\eta u_{0}\right], \\
\eta u_{0} h-u_{0}^{2} & =-q=+\frac{1}{R e}\left[4 \rho h^{\prime \prime}+4 h^{\prime}-\frac{h}{\rho}+\eta^{2} h-2 g^{\prime}+\frac{g}{\rho}-\eta f-\eta u_{0}\right],
\end{array}\right\}
$$

where the prime denotes the derivative with respect to $\rho$. The no-slip conditions yield

$$
f(1)=g(1)=h(1)=0,
$$

and we require all physical properties to be finite throughout the cross-section. By calculating the Frobenius expansion of (3.9) about $\rho=0$, we find the finiteness conditions amount to

$$
\begin{gathered}
g(0)-h(0)=0, \\
\left(\frac{\eta^{2}}{R e}-2 \eta\right) f(0)+4 g(0)-\eta q(0)+\frac{8}{R e} f^{\prime}(0)=\frac{12}{R e}, \\
\frac{2 \eta}{3 R e} f(0)+\left(2 \eta-\frac{\eta^{2}}{R e}\right) g(0)+q(0)-\frac{8}{3 R e} h^{\prime}(0)=4-\frac{2 \eta}{R e} .
\end{gathered}
$$

For small $\eta$ the solution of (3.9) is approximated (to first order) by

$$
\left.\begin{array}{l}
f=f_{0}+\eta f_{1}+O\left(\eta^{2}\right), \\
g=g_{0}+\eta g_{1}+O\left(\eta^{2}\right), \\
h=h_{0}+\eta h_{1}+O\left(\eta^{2}\right), \\
q=q_{0}+\eta q_{1}+O\left(\eta^{2}\right) .
\end{array}\right\}
$$


The leading-order terms give the Dean solution:

$$
\begin{aligned}
& f_{0}=-\frac{3}{2}(1-\rho)+\frac{R e^{2}}{1440}(1-\rho)\left(19-21 \rho+9 \rho^{2}-\rho^{3}\right), \\
& g_{0}=\frac{R e}{72}(1-\rho)^{2}(4-\rho), \\
& h_{0}=\frac{R e}{72}(1-\rho)\left(4-23 \rho+7 \rho^{2}\right), \\
& q_{0}=\frac{1}{3}\left(9-6 \rho+2 \rho^{2}\right) .
\end{aligned}
$$

The first-order terms are

$$
\begin{aligned}
f_{1}= & -\frac{\operatorname{Re}^{3}}{1814400}(\rho-1)\left(-2969+4381 \rho-3249 \rho^{2}+1301 \rho^{3}-274 \rho^{4}+20 \rho^{5}\right) \\
& +\frac{\operatorname{Re}}{144}(\rho-1)\left(29+5 \rho-3 \rho^{2}\right), \\
g_{1}= & \frac{\operatorname{Re}^{2}}{8640}(\rho-1)^{2}\left(-13+15 \rho-7 \rho^{2}+\rho^{3}\right)-\frac{1}{3}(\rho-1)^{2}, \\
h_{1}= & \frac{\operatorname{Re}^{2}}{8640}(\rho-1)\left(13-224 \rho+266 \rho^{2}-124 \rho^{3}+17 \rho^{4}\right)+\frac{1}{6}(\rho-1)(2-\rho), \\
q_{1}= & \frac{\operatorname{Re}}{2160}\left(-101+120 \rho-90 \rho^{2}+30 \rho^{3}-3 \rho^{4}\right)+\frac{1}{3 \operatorname{Re}}(1-3 \rho) .
\end{aligned}
$$

As $\eta$ increases, the series solution (3.10) loses accuracy and a numerical solution is needed; this is described at the end of this subsection.

Now consider the $O\left(\epsilon^{2}\right)$ equations. From Germano's work we know that, at leading order, torsion does not affect the flow in pipes with circular cross-section. However, once curvature is present, torsion has an effect. We wish to include torsion in the model at low order, so we assume that $\epsilon^{-1} \gg \lambda \gg 1$ and

$$
\begin{array}{cc}
u_{2}=\lambda\left(u_{20}+O\left(\lambda^{-1}\right)\right), & v_{2}=\lambda\left(v_{20}+O\left(\lambda^{-1}\right)\right), \\
w_{2}=\lambda\left(w_{20}+O\left(\lambda^{-1}\right)\right), & p_{2}=\lambda\left(p_{20}+O\left(\lambda^{-1}\right)\right) .
\end{array}
$$

It follows that the next terms in our Dean-type expansion are $O\left(\epsilon^{2} \lambda\right)$. These terms depend explicitly upon $s$ via the factor $\left(\epsilon^{2} \lambda\right)_{s} / \epsilon^{2} \lambda$ (see the Appendix). We assume that $\epsilon^{2} \lambda=\kappa_{0} \tau_{0} e^{\beta s}$ (so that $\left(\epsilon^{2} \lambda\right)_{s} / \epsilon^{2} \lambda=\beta$ ); this is a local approximation to pipes with more general non-uniformities, by the same argument as before.

We look for solutions of the form

$$
u_{20}=r F(\rho) \cos \xi, \quad v_{20}=G(\rho) \cos \xi, \quad w_{20}=H(\rho) \sin \xi, \quad p_{20}=r Q(\rho) \cos \xi .
$$

This yields the following system of coupled linear ordinary differential equations:

$$
\begin{gathered}
\beta F-f+2 G^{\prime}+\frac{H+G}{\rho}=0 \\
2 \beta F(1-\rho)-4 G-2(1-\rho) f=-\beta Q+q+\frac{1}{\operatorname{Re}}\left[4 \rho F^{\prime \prime}+8 F^{\prime}\right. \\
\left.-2 \beta G^{\prime}-\frac{\beta G}{\rho}-\frac{\beta H}{\rho}+2 g^{\prime}+\frac{g}{\rho}-\frac{h}{\rho}\right],
\end{gathered}
$$




$$
\begin{aligned}
2 \beta G(1-\rho)-2(1-\rho) g= & -2 \rho Q^{\prime}-Q+\frac{1}{\operatorname{Re}}\left[-2 \beta \rho F^{\prime}-\beta F+\beta^{2} G-\frac{G}{\rho}\right. \\
& \left.-2 H^{\prime}-\frac{H}{\rho}+2 \rho f^{\prime}+f-\beta \underline{\underline{-\eta g}}+2(1-\rho)\right], \\
2 \beta H(1-\rho)+2(1-\rho) h= & Q+\frac{1}{\operatorname{Re}}\left[\beta F+2 G^{\prime}-\frac{G}{\rho}+4 \rho H^{\prime \prime}\right. \\
& +4 H^{\prime}+\beta^{2} H-\frac{H}{\rho}-f+\beta \underline{\underline{+\eta h}-2(1-\rho)] .}
\end{aligned}
$$

(The significance of the underlined terms is discussed below.) The boundary conditions are

$$
\begin{gathered}
F(1)=G(1)=H(1)=0, \\
G(0)+H(0)=0, \\
\frac{2 \beta}{3} F(0)+\left(2 \beta \operatorname{Re}-\beta^{2}\right) G(0)+\operatorname{Re} Q(0)+\frac{8}{3} H^{\prime}(0)=\frac{2}{3} f(0)+(2 \operatorname{Re}-\beta) g(0) \underline{-\eta g(0)}+2, \\
\left(2 \beta \operatorname{Re}-\beta^{2}\right) F(0)-4 \operatorname{ReG}(0)+\beta \operatorname{Re} Q(0)-8 F^{\prime}(0)=(2 \operatorname{Re}-\beta) f(0) \underline{-\eta f(0)}+\operatorname{Re} q(0) .
\end{gathered}
$$

For small $\beta$ equations (3.11)-(3.14) can be solved via an expansion similar to (3.10), i.e.

$$
F=F_{0}+\beta F_{1}+O\left(\beta^{2}\right), \quad \text { etc. }
$$

At this stage, we must consider the question of how valid these expansions are. For example,

$$
u=u_{0}+\kappa_{0} \mathrm{e}^{\eta s}\left(r f_{0}+\eta r f_{1}+O\left(\eta^{2}\right)\right)+\kappa_{0} \tau_{0} \mathrm{e}^{\beta s}\left(r F_{0}+\beta r F_{1}+O\left(\beta^{2}\right)\right)+O\left(\epsilon^{2}\right),
$$

and therefore important terms might be inadvertently omitted unless $\eta$ is sufficiently small. If $\epsilon^{2} \lambda \gg \epsilon \eta$ then the expansion is valid; otherwise, we solve the governing ordinary differential equations numerically.

First, suppose that $\epsilon^{2} \lambda \gg \epsilon \eta$. Then the underlined terms in (3.11)-(3.14) and the boundary conditions can be neglected, and the leading-order solutions for the $O\left(\epsilon^{2} \lambda\right)$ terms are

$$
F_{0}=-f_{1}, \quad G_{0}=-g_{1}, \quad H_{0}=h_{1}, \quad Q_{0}=-q_{1} .
$$

The first-order terms are found to be

$$
\begin{aligned}
F_{1}= & \frac{\operatorname{Re}^{4}}{4877107200}(\rho-1)\left(-697301+1162699 \rho-1065233 \rho^{2}+610567 \rho^{3}-232037 \rho^{4}\right. \\
& \left.+56083 \rho^{5}-7757 \rho^{6}+415 \rho^{7}\right) \\
& -\frac{R e^{3}}{4877107200}(\rho-1)\left(109335-159759 \rho+116909 \rho^{2}-47241 \rho^{3}+11559 \rho^{4}\right. \\
& \left.-1671 \rho^{5}+93 \rho^{6}\right) \\
& -\frac{R e^{2}}{172800}(\rho-1)\left(-3784+3181 \rho-889 \rho^{2}-139 \rho^{3}+41 \rho^{4}\right) \\
& +\frac{\operatorname{Re}}{138240}(\rho-1)\left(-117+123 \rho-47 \rho^{2}+3 \rho^{3}\right) \\
& +\frac{1}{48}(\rho-1)(\rho-6),
\end{aligned}
$$




$$
\begin{aligned}
G_{1}= & \frac{\operatorname{Re}^{3}}{203212800}(\rho-1)^{2}\left(11264+1647 \rho-6990 \rho^{2}+4463 \rho^{3}-1234 \rho^{4}+125 \rho^{5}\right) \\
& +\frac{\operatorname{Re}^{2}}{58060800}(\rho-1)^{2}\left(6407-6948 \rho+3147 \rho^{2}-758 \rho^{3}+62 \rho^{4}\right) \\
& +\frac{\operatorname{Re}}{2880}(\rho-1)^{2}\left(101+19 \rho-8 \rho^{2}\right) \\
& -\frac{1}{2304}(\rho-1)^{2}(\rho-8), \\
H_{1}= & -\frac{\operatorname{Re}^{3}}{203212800}(\rho-1)\left(-11264-281149 \rho+537151 \rho^{2}-458039 \rho^{3}+205911 \rho^{4}\right. \\
- & \left.48105 \rho^{5}+4115 \rho^{6}\right) \\
+ & \frac{\operatorname{Re}}{58060800}(\rho-1)\left(6407-52879 \rho+64371 \rho^{2}-33629 \rho^{3}+8896 \rho^{4}-806 \rho^{5}\right) \\
+ & \frac{\operatorname{Re}}{2880}(\rho-1)\left(101+132 \rho-73 \rho^{2}+12 \rho^{3}\right) \\
+ & \frac{1}{2304}(\rho-1)\left(8-43 \rho+7 \rho^{2}\right), \\
Q_{1}= & \frac{\operatorname{Re}}{3628800}\left(2027-5460 \rho+5740 \rho^{2}-3500 \rho^{3}+1260 \rho^{4}-252 \rho^{5}+20 \rho^{6}\right) \\
& \quad-\frac{\operatorname{Re}}{3628800}\left(4421-5985 \rho+4200 \rho^{2}-1575 \rho^{3}+315 \rho^{4}-21 \rho^{5}\right) \\
& +\frac{1}{288 \operatorname{Re}}\left(79-54 \rho+18 \rho^{2}\right)-\frac{1}{360}\left(278-255 \rho+110 \rho^{2}-25 \rho^{3}\right) .
\end{aligned}
$$

An important feature of these analytic results is that they all increase with the Reynolds number, $R e$, which will eventually lead to the breakdown of the series expansion. The results presented above have not been given in terms of a Dean number due to the non-uniformity of the curvature parameter. In the present model $\epsilon=\kappa_{0} \mathrm{e}^{\eta s}$ so, assuming that $\epsilon \ll 1$ for a suitable range of $s$, we can define a slowly varying pseudo-Dean number $D(s)=2 \kappa_{0} \mathrm{e}^{\eta s} R^{2}$. For $\eta=0, D(s)$ is the Dean number (as defined by Dean 1928). Dean found that the expansion is invalid if the Dean number exceeds 576 .

Some of the different centrelines that can be created using the various parameters are as follows (unless otherwise stated the parameters are assumed to be non-zero):

(a) torus, $\left(\tau_{0}=\eta=0\right)$;

(b) helix, $(\eta=\beta=0)$;

(c) spiral, $\left(\tau_{0}=0\right)$;

(d) spiral with uniform torsion, $(\beta=\eta \neq 0)$;

(e) 'stretched' helix, $(\eta=0)$;

$(f)$ a pipe with curvature inversely proportional to torsion, $(\beta=0)$.

Figure 2 shows pipes with these centrelines. For pipes $(d)$ and $(f)$ the assumption that $\epsilon^{2} \lambda \gg \epsilon \eta$ is too restrictive. Therefore we have chosen to solve the $O(\epsilon)$ and $O\left(\epsilon^{2} \lambda\right)$ equations (3.9) and (3.11)-(3.14) numerically, using the collocation method. (The NAG library subroutine d02tgf was used.) This method is described in many advanced texts on numerical methods (e.g. Gerald \& Wheatley 1994). The numerical results have been checked with the analytical results for $(a),(b),(c)$, and $(e)$, and 
(a)

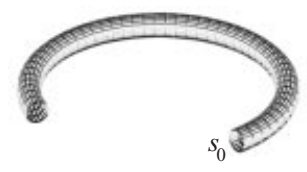

(d)

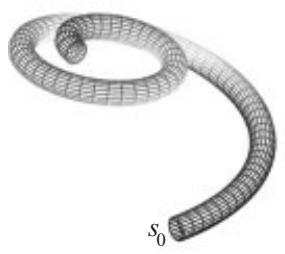

D. Gammack and P. E. Hydon

(b)

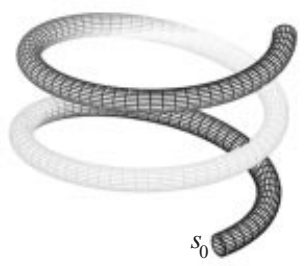

(e)

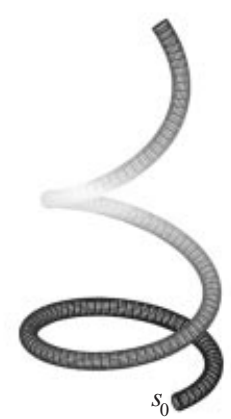

(c)

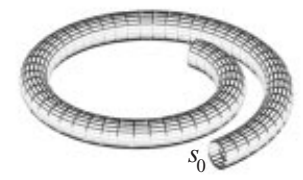

$(f)$

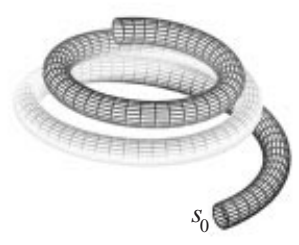

Figure 2. Pipe shapes $(a)$ torus, $(b)$ helix, $(c)$ spiral, $(d)$ spiral with uniform torsion, $(e)$ 'stretched helix', $(f)$ pipe with curvature inversely proportional to torsion.
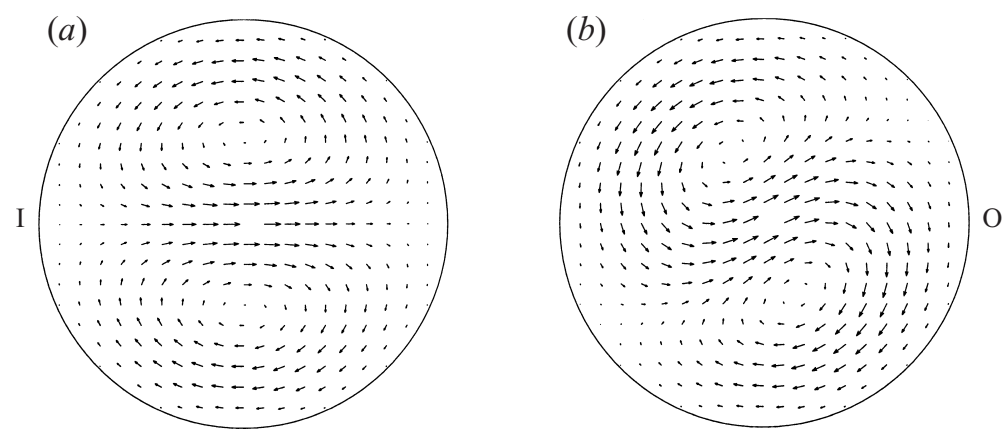

FiguRE 3. Secondary velocity vector field plots: $(a)$ Dean flow, $\kappa_{0}=0.09, \tau_{0}=0.0 ;(b)$ helical flow, $\kappa_{0}=0.09, \tau_{0}=0.4$. I and $\mathrm{O}$ denote respectively the innermost and outermost parts of the pipe wall.

have been shown to be accurate. (Results obtained from the numerical method have (Numerical) in the figure caption.)

\subsection{Results}

Figure 3 shows how uniform torsion breaks the secondary flow symmetry of toroidal flow. It is not possible to define a stream function, and therefore all secondary velocity fields are displayed by vector field plots. Dean flow and helical flow are discussed by other authors (see the reviews by Berger, Talbot \& Yao 1983 and Tuttle 1990).

We first consider a spiral pipe (figure $2 c$ ) whose centreline has zero torsion and exponentially increasing curvature (figures 4 to 6 ). The secondary flow patterns for 

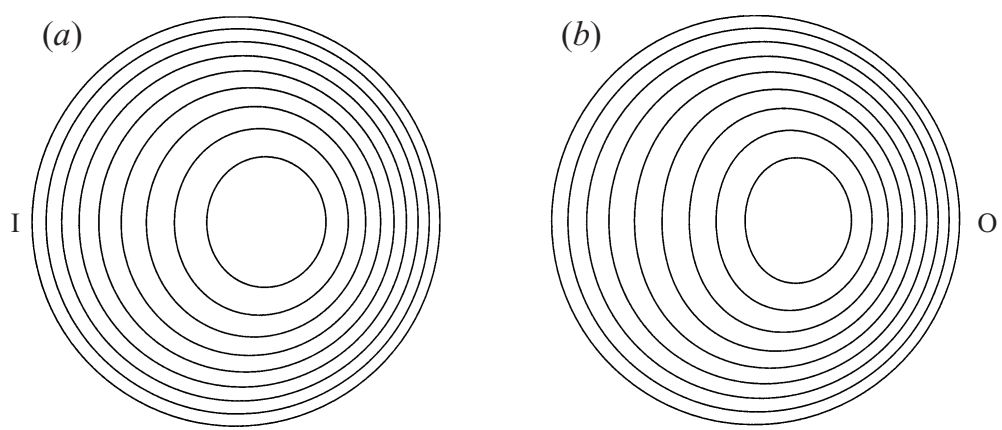

FIGURE 4. The axial flow for a pipe with a spiral centreline: $R e=30, \kappa_{0}=0.01, \eta=0.1$ and (a) $s=0.001 ;(b) s=15$
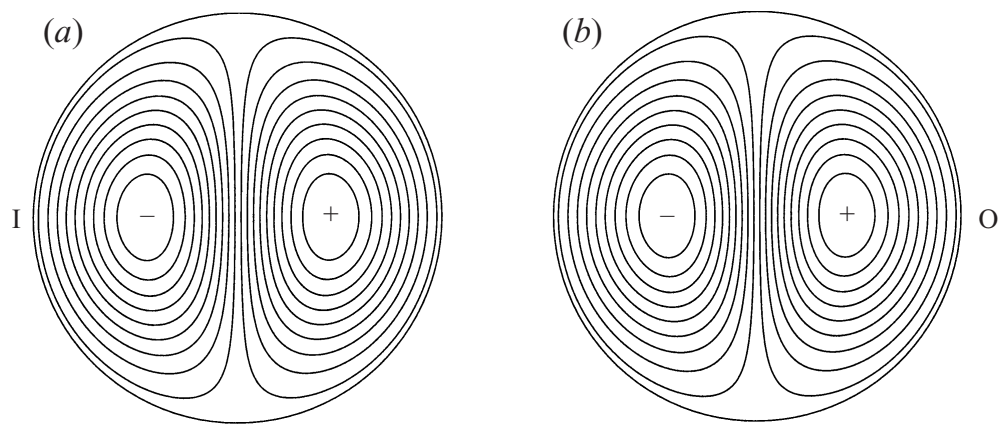

FIGURE 5. The axial perturbation to: $(a)$ Poiseuille flow, $(b)$ Dean flow $(+$ denotes a perturbation in the direction of the mean flow, - denotes a perturbation in the opposite direction). Parameters as for figure 4.

the spiral look exactly the same as Dean flow. This similarity arises because increasing the curvature merely increases the magnitude of the vector field (to leading order). Figure 4 shows contour plots of the axial velocity at two positions along the pipe. As the fluid travels along the pipe the peak axial velocity is forced towards the outer wall by the increasing curvature. In Poiseuille flow the contours of axial velocity are concentric circles, and the perturbation forces these circles towards the outer wall. The axial perturbations to Poiseuille flow and Dean flow (figure 5) consist of two cells, with opposing flows. As the curvature increases so does the magnitude of the cells and hence the peak axial velocity is forced to the outer wall; as the curvature becomes even larger we would expect to see a deformation of the circles. A similar result is seen in high Dean number toroidal flow, as shown by Berger et al. The amplitude of the sinusoidal perturbation to the wall shear stress (WSS) increases with increasing $s$, and the position of least WSS occurs at the inner wall (figure 6). (In all plots of the WSS we show the axial and azimuthal components and the root-mean-squared (total).)

For a pipe with a spiral centreline and uniform torsion (figure $2 d$ ) the results are similar to those seen in helical flow. The addition of uniform torsion to flow in a spiral pipe skews the secondary flow vortices (figure 7), breaking the symmetry, and moves the position of maximum axial velocity (figure 8 ). The sinusoidal perturbation to the azimuthal WSS has an increasing effect on the total WSS as the flow progresses along the pipe, causing a 'bump' which reduces the region of low WSS (see figure 9). This is because the sinusoidal perturbation to the azimuthal WSS increases in magnitude 

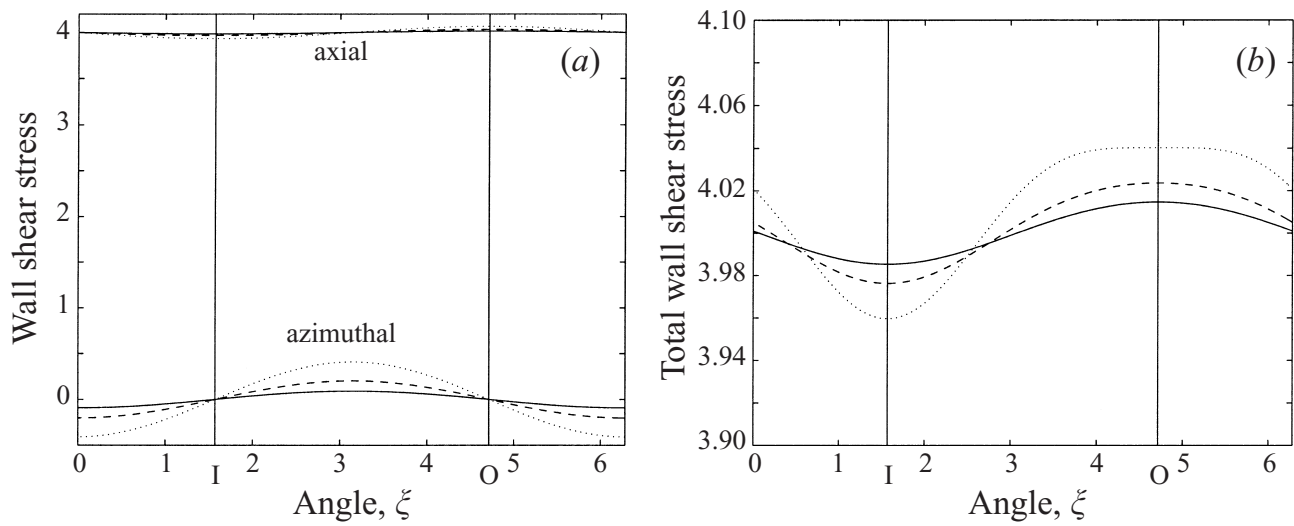

FIGURE 6. The wall shear stress (WSS) for a pipe with a spiral centreline:,$- s=0.001$; - - $s=8 ; \cdots, s=15$. (a) Axial and azimuthal WSS; $(b)$ total WSS. Parameters as for figure 4.
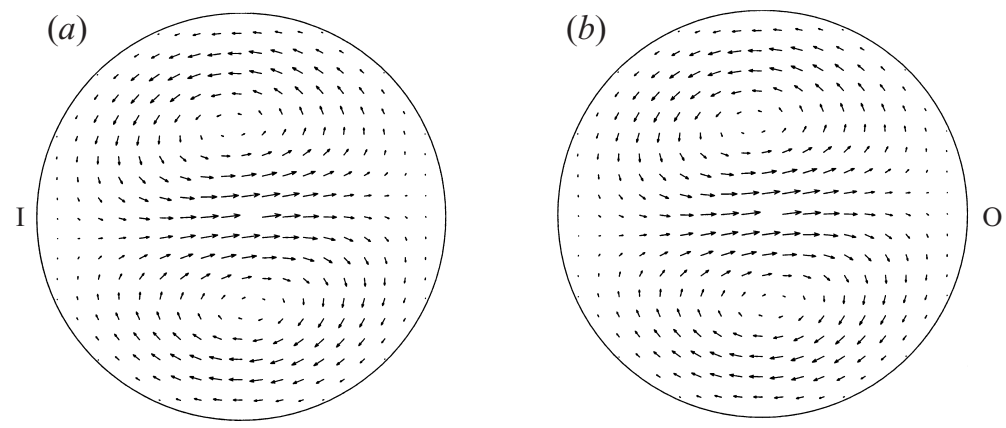

FIgURE 7. (Numerical) The secondary velocity vector field for a spiral pipe with uniform torsion: $R e=30, \kappa_{0}=0.09, \eta=0.1, \tau_{0}=0.1, \beta=0.1$ and $(a) s=0.001 ;(b) s=5$.
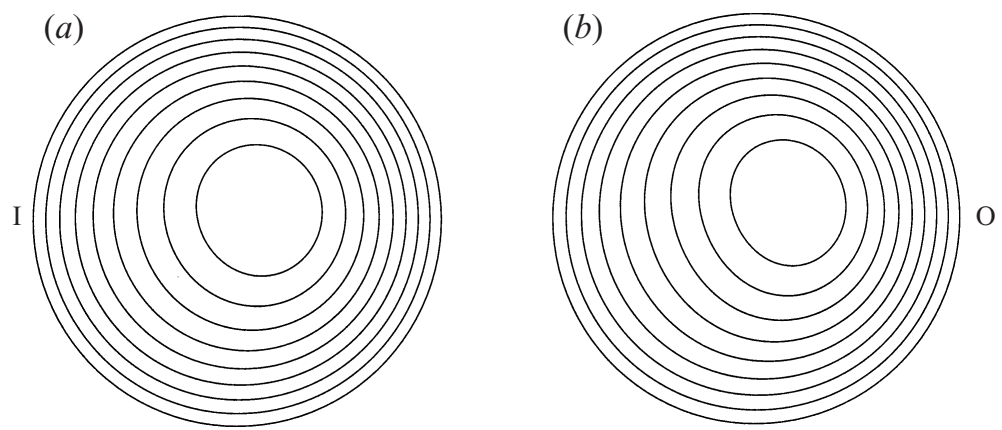

FIGURE 8. (Numerical) The axial velocity for a spiral pipe with uniform torsion: parameters as for figure 7.

more rapidly than the axial WSS, due to the increasing magnitude of the secondary velocity vector field, which in turn is caused by the increasing curvature.

For the 'stretched' helix (figure $2 e$ ) the effects seen in helical flow are exaggerated as the fluid moves further along the pipe (figure 10). The secondary flow vortices become more skewed, and the mechanisms for this are shown in the secondary flow perturbation plots (figure 11). The perturbation to Dean flow breaks the symmetry, 

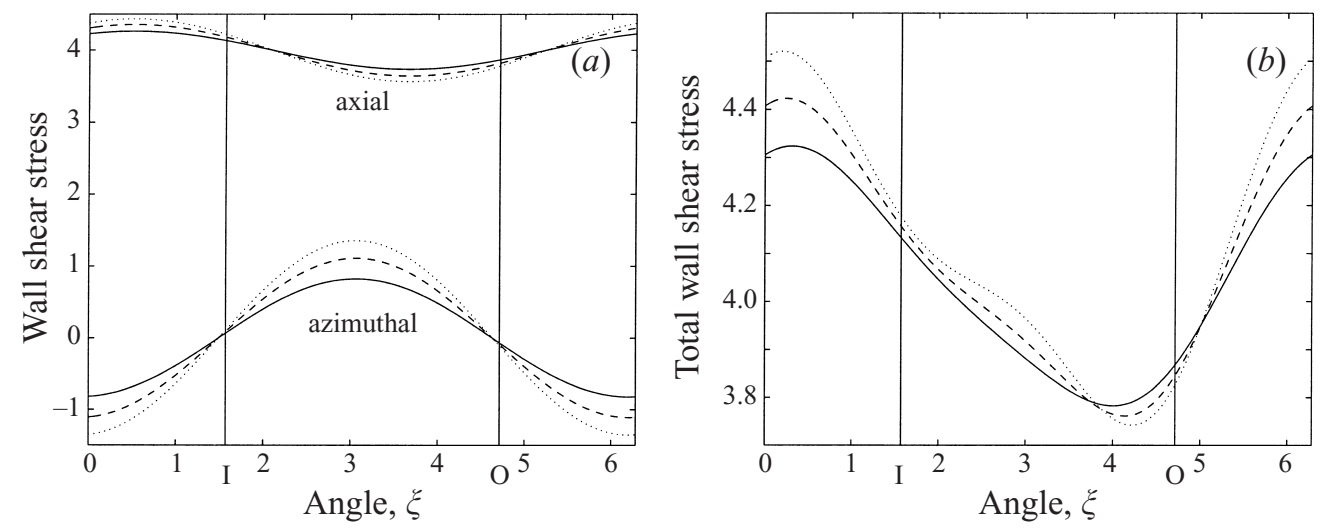

FIgURE 9. (Numerical) The wall shear stress for a spiral pipe with uniform torsion:,$- s=0.001$; ,$--- s=3 ; \cdots \cdots, s=5$. (a) Axial and azimuthal WSS; $(b)$ total WSS. Parameters as for figure 7.
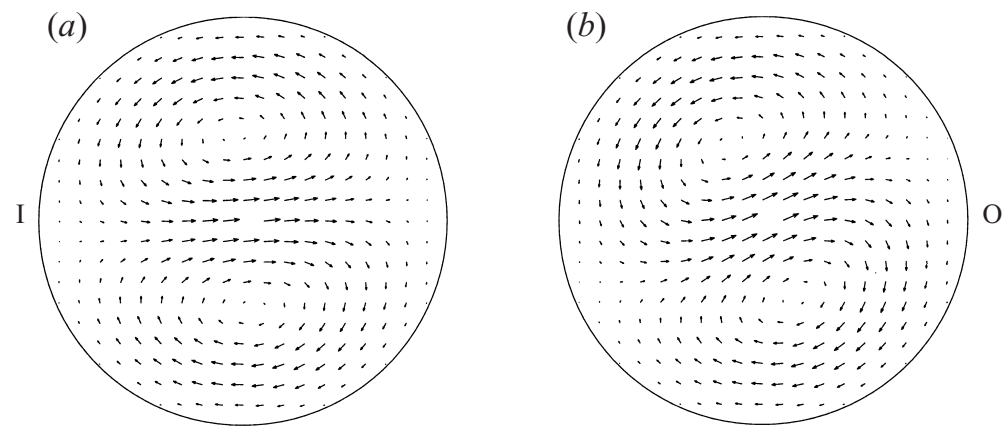

FIGURE 10. The secondary flow for a helical pipe with increasing torsion: $R e=25, \kappa_{0}=0.09$, $\eta=0, \tau_{0}=0.1, \beta=0.1$ and $(a) s=0.001 ;(b) s=15$.

and the perturbation to helical flow increases the skew. The contour plots of axial velocity (figure 12) demonstrate the same effect of torsion. The axial perturbations to Dean flow and helical flow (figure 13) both consist of two cells, with opposing flows, and demonstrate the mechanism for the increased skew in the axial velocity. Figure 14 shows the effect on the WWS: as torsion increases there is a phase shift in the direction of the torsion. This phase shift is more prominent in the axial component because the coefficient of the $O\left(\epsilon^{2} \lambda \beta\right)$ term is larger than that for the azimuthal component.

Finally we consider a pipe for which torsion is inversely proportional to curvature (figure $2 f$ ). Here torsion is dominant at the beginning of the pipe, but as the arclength increases, curvature has a more significant effect on the flow. This is shown in the secondary velocity plots (figure 15) where the two-vortex structure skews towards Dean flow as $s$ increases. Figure 16 shows that there is no noticeable effect on the peak axial velocity. The WSS produces a result that at first seems counter-intuitive: the maximum WSS occurs at the inner wall (figure 17). The same phenomenon is seen in Dean flow at very low Reynolds numbers (see Larrain \& Bonilla 1970; Murata et al. 1976). It is due to the geometrical effect of curvature upon the basic Poiseuille flow, which is dominant if the convective inertia is sufficiently small. 

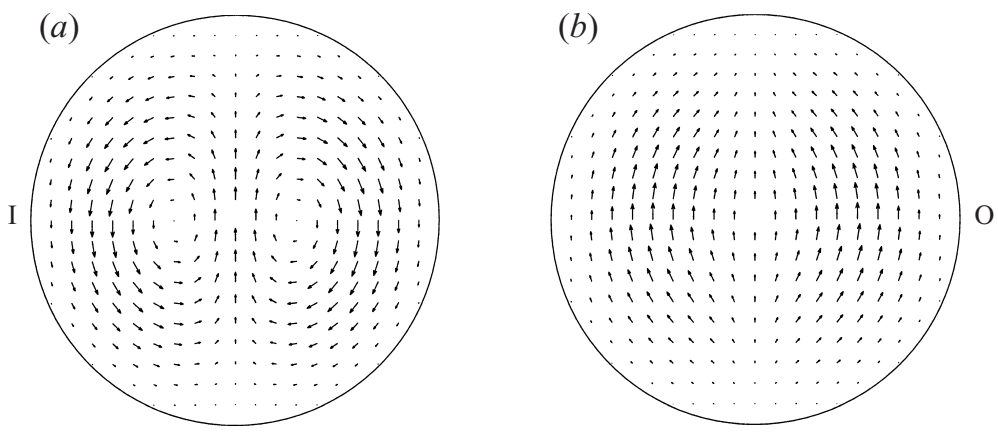

FIGURE 11. The perturbations to the secondary flow for a helical pipe with increasing torsion: (a) perturbation to Dean flow; (b) perturbation to helical flow. Parameters as for figure 10.
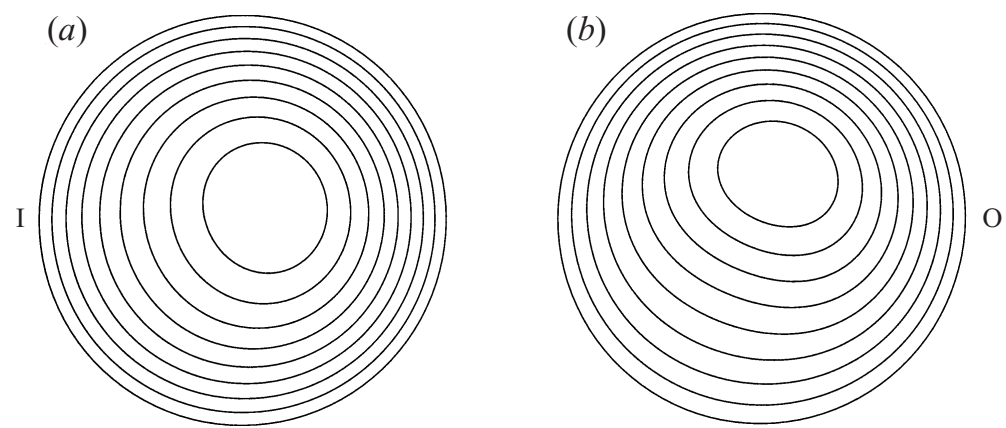

FIGURE 12. The axial flow for a helical pipe with increasing torsion: parameters as for figure 10 with $(a) s=0.001 ;(b) s=15$.
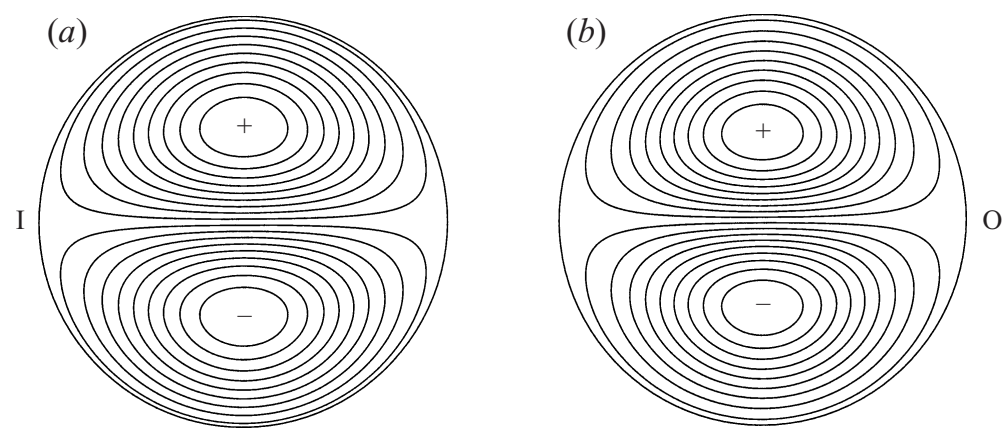

FIGURE 13. The perturbations to the axial flow for a helical pipe with increasing torsion:

(a) perturbation to Dean flow; (b) perturbation to helical flow. Parameters as for figure 10.

\section{Unsteady flow}

Following Hydon (1994) the Navier-Stokes equations are expressed in terms of the non-dimensionalized variables

$$
\left.\begin{array}{rl}
(u, v, w) & =\left(\frac{\Omega}{K} \hat{u}, \frac{a}{v} \hat{v}, \frac{a}{v} \hat{w}\right), \quad(s, r)=\left(\frac{\gamma}{a} \hat{s}, a^{-1} \hat{r}\right), \quad \text { where } \quad \gamma=\frac{v \Omega}{a K}, \\
p & =-\frac{K a^{2}}{v^{2}} \hat{s} \cos (\Omega \hat{t})+\frac{a^{2}}{v^{2} \hat{\rho_{0}}} \hat{p}, \quad t=\frac{\Omega}{2 \pi} \hat{t}, \quad \epsilon=a \hat{\kappa}, \quad
\end{array}\right\}
$$



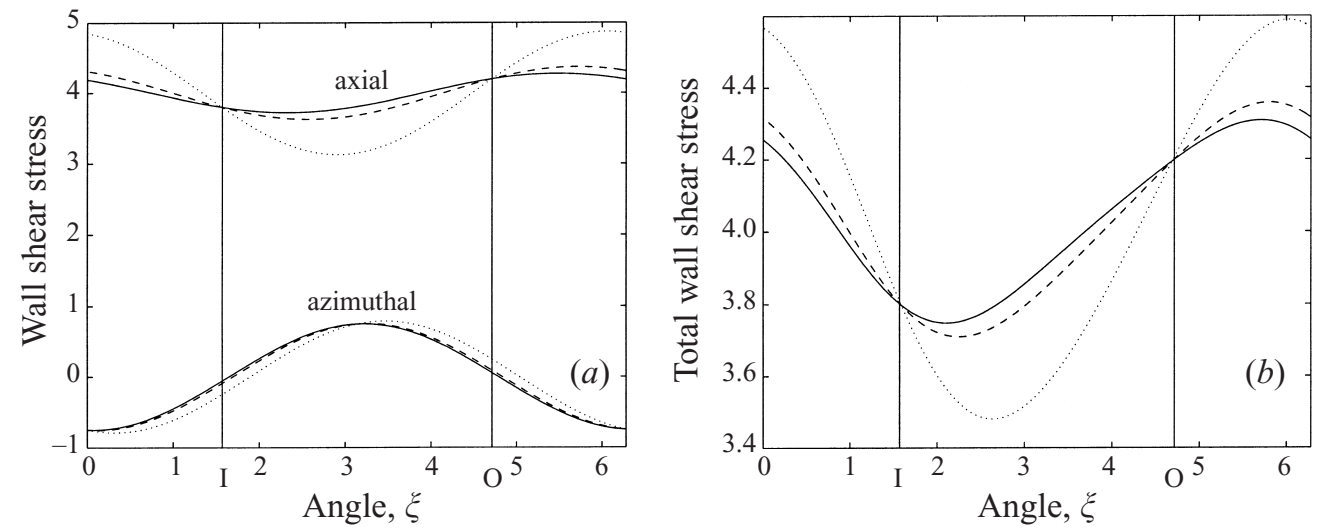

FIGURE 14. The wall shear for a helical pipe with increasing torsion:,$- s=0.001 ;---, s=8$; $\cdots \cdots, s=15$. (a) Axial and azimuthal WSS; $(b)$ total WSS. Parameters as for figure 10.
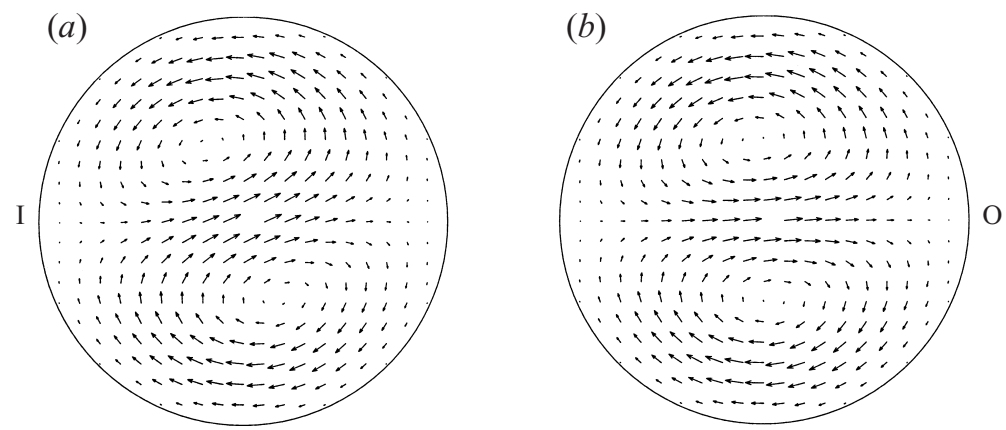

FIGURE 15. (Numerical) The secondary velocity vector field for a pipe with torsion inversely proportional to curvature: $\operatorname{Re}=10, \kappa_{0}=0.01, \eta=0.3, \tau_{0}=0.31, \beta=0.0$ and $(a) s=0.001$; (b) $s=5$.
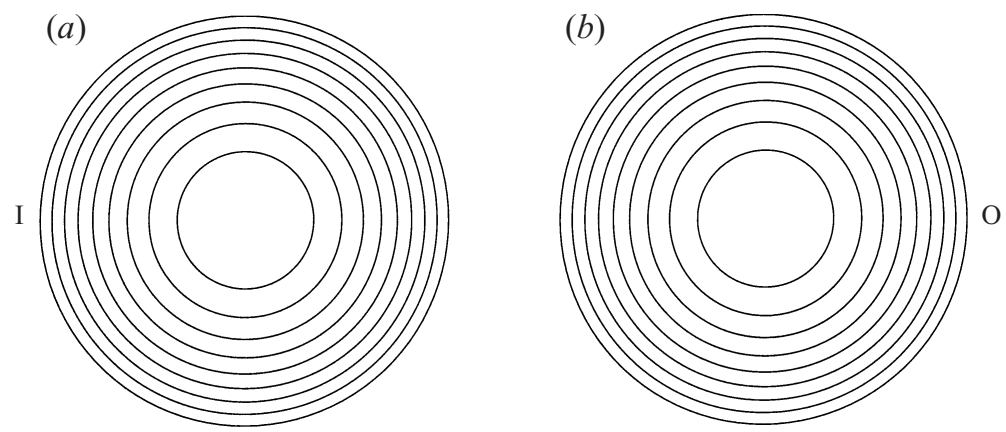

FIgURE 16. (Numerical) The axial velocity for a pipe with parameters as figure 15 with (a) $s=0.001 ;(b) s=5$.

where $K$ is the amplitude and $\Omega$ is the frequency of the pressure pulse, with $(v, a)$ as before. We find that four dimensionless parameters govern the flow: the frequency (Womersley) parameter, $\alpha=\sqrt{a^{2} \Omega / v}$, the curvature, $\epsilon(s)$, the ratio of torsion to curvature, $\lambda(s)$, and $G_{0}=K^{2} a^{2} /\left(\Omega^{2} v^{2}\right)$. Hydon (1994) and Mullin \& Greated (1980) use the parameter $G=2 \epsilon_{0} G_{0}$, where $\epsilon_{0}$ is the curvature in a uniformly curved pipe. Mullin \& Greated showed that in the low-frequency limit $G$ is equivalent to the Dean 

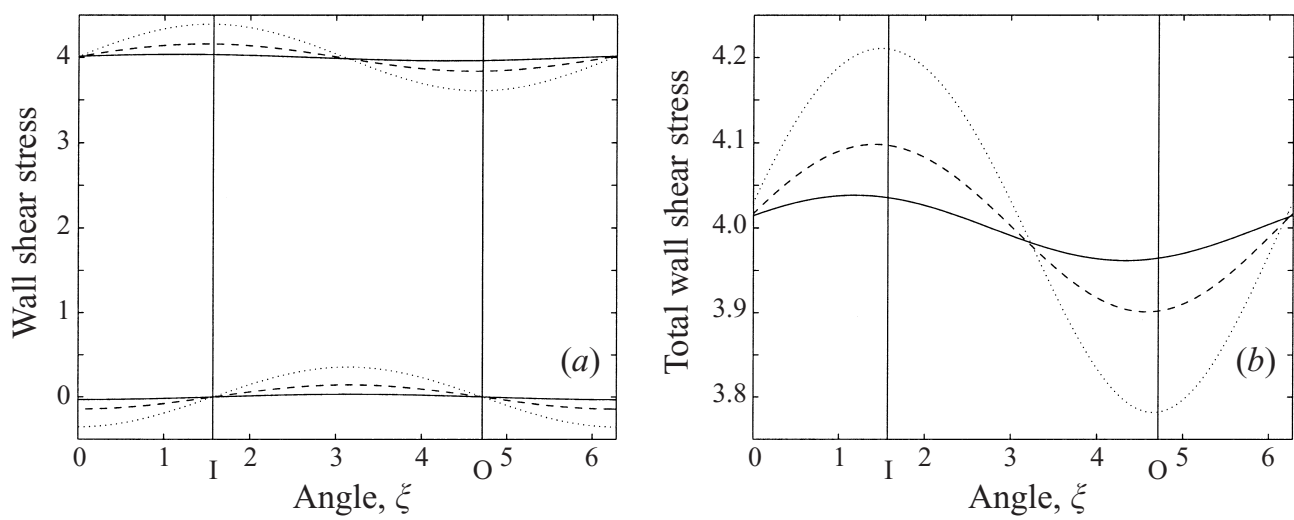

Figure 17. (Numerical) The wall shear stress for a pipe with torsion inversely proportional to curvature:,$- s=0.001 ;-\cdots, s=3 ; \cdots \cdots, s=5$. (a) Axial and azimuthal WSS; $(b)$ total WSS. Parameters as for figure 15.

parameter. The Navier-Stokes equations for incompressible unsteady flow are

$$
\begin{gathered}
\varpi\left(u_{s}-\epsilon \lambda u_{\xi}\right)+v_{r}+\frac{1}{r} w_{\xi}+\frac{1}{r} v+\epsilon \varpi(v \sin \xi+w \cos \xi)=0, \\
\frac{\alpha^{2}}{2 \pi} u_{t}+\varpi u\left(u_{s}-\epsilon \lambda u_{\xi}\right)+v u_{r}+\frac{1}{r} w u_{\xi}+\epsilon \varpi u(v \sin \xi+w \cos \xi) \\
=-\frac{\varpi}{G_{0}}\left(p_{s}-\epsilon \lambda p_{\xi}\right)+\alpha^{2} \varpi \cos 2 \pi t+\left(\frac{\partial}{\partial r}+\frac{1}{r}\right)\left(u_{r}+\epsilon \varpi u \sin \xi\right) \\
+\frac{1}{r} \frac{\partial}{\partial \xi}\left(\frac{1}{r} u_{\xi}+\epsilon \varpi u \cos \xi\right) \\
-\frac{1}{G_{0}}\left(\left(\frac{\partial}{\partial r}+\frac{1}{r}\right)\left(\varpi\left(v_{s}-\epsilon \lambda v_{\xi}\right)\right)+\frac{1}{r} \frac{\partial}{\partial \xi}\left(\varpi\left(w_{s}-\epsilon \lambda w_{\xi}\right)\right)\right), \\
\frac{\alpha^{2}}{2 \pi} v_{t}+\varpi u\left(v_{s}-\epsilon \lambda v_{\xi}\right)+v v_{r}+\frac{1}{r} w v_{\xi}-\frac{1}{r} w^{2}-\epsilon \varpi G_{0} u^{2} \sin \xi \\
=-p_{r}-\left(\frac{1}{r} \frac{\partial}{\partial \xi}+\epsilon \varpi \cos \xi\right)\left(w_{r}+\frac{1}{r} w-\frac{1}{r} v_{\xi}\right) \\
+\varpi\left(\frac{\partial}{\partial s}-\epsilon \lambda \frac{\partial}{\partial \xi}\right)\left(\frac{\varpi}{G_{0}}\left(v_{s}-\epsilon \lambda v_{\xi}\right)-u_{r}-\epsilon \varpi u \sin \xi\right), \\
\frac{\alpha^{2}}{2 \pi} w_{t}+\varpi u\left(w_{s}-\epsilon \lambda w_{\xi}\right)+v w_{r}+\frac{1}{r} w w_{\xi}+\frac{1}{r} v w-\epsilon \varpi G_{0} u^{2} \cos \xi \\
=-\frac{1}{r} p_{\xi}+\left(\frac{\partial}{\partial r}+\epsilon \varpi \sin \xi\right)\left(w_{r}+\frac{1}{r} w-\frac{1}{r} v_{\xi}\right) \\
-\varpi\left(\frac{\partial}{\partial s}-\epsilon \lambda \frac{\partial}{\partial \xi}\right)\left(\frac{1}{r} u_{\xi}+\epsilon \varpi u \cos \xi-\frac{\varpi}{G_{0}}\left(w_{s}-\epsilon \lambda w_{\xi}\right)\right) .
\end{gathered}
$$




\subsection{The solution}

As with the steady flow case we assume that $\epsilon \ll 1$ and construct a small-parameter perturbation, i.e.

$$
\left.\begin{array}{rl}
u & =u_{0}(r, t)+\epsilon(s) u_{1}(r, s, t) \sin (\xi)+\epsilon(s)^{2} u_{2}(r, s, \xi, t)+O\left(\epsilon(s)^{3}\right), \\
v & =\epsilon(s) v_{1}(r, s, t) \sin (\xi)+\epsilon(s)^{2} v_{2}(r, s, \xi, t)+O\left(\epsilon(s)^{3}\right), \\
w & =\epsilon(s) w_{1}(r, s, t) \cos (\xi)+\epsilon(s)^{2} w_{2}(r, s, \xi, t)+O\left(\epsilon(s)^{3}\right), \\
p & =p_{0}(s, t)+\epsilon(s) p_{1}(r, s, t) \sin (\xi)+\epsilon(s)^{2} p_{2}(r, s, \xi, t)+O\left(\epsilon(s)^{3}\right),
\end{array}\right\}
$$

where we follow Lyne (1971) in assuming that $p_{0 s}=-\alpha^{2} \cos 2 \pi t$. The boundary conditions are $u=v=w=0$ at $r=1$. We also require that the velocities and stresses are finite everywhere in the cross-section.

The leading-order (straight pipe) solution was solved by Sexl (1930):

$$
u_{0}=u_{0 R} \cos 2 \pi t-u_{0 I} \sin 2 \pi t,
$$

where

$$
\begin{aligned}
& u_{0 R}=\operatorname{Re}\left(-i+i \frac{J_{0}((1-i) \alpha r / \sqrt{2})}{J_{0}((1-i) \alpha / \sqrt{2})}\right), \\
& u_{0 I}=\operatorname{Im}\left(-i+i \frac{J_{0}((1-i) \alpha r / \sqrt{2})}{J_{0}((1-i) \alpha / \sqrt{2})}\right) .
\end{aligned}
$$

The $O\left(\epsilon^{1}\right)$ terms are

$$
\begin{gathered}
v_{1, r}+\frac{1}{r}\left(v_{1}-w_{1}\right)=-\eta u_{1}, \\
u_{1, r r}+\frac{1}{r} u_{1, r}-\frac{1}{r^{2}} u_{1}-\frac{\alpha^{2}}{2 \pi} u_{1, t}-u_{0, r} v_{1}= \\
\alpha^{2} r \cos 2 \pi t-u_{0, r}+\eta u_{0} u_{1} \\
+\frac{\eta}{G_{0}}\left(v_{1, r}+\frac{1}{r}\left(v_{1}-w_{1}\right)+p_{1}\right), \\
\frac{\alpha^{2}}{2 \pi} v_{1, t}+\frac{1}{r^{2}}\left(v_{1}-w_{1}\right)-\frac{1}{r} w_{1, r}+p_{1, r}=G_{0} u_{0}^{2}+\eta\left(-u_{0}+u_{1, r}-u_{0} v_{1}\right)-\frac{\eta^{2}}{G_{0}} v_{1}, \\
-\frac{\alpha^{2}}{2 \pi} w_{1, t}+w_{1, r}+\frac{1}{r} w_{1, r}+\frac{1}{r^{2}}\left(v_{1}-w_{1}\right)-\frac{1}{r} v_{1, r}-\frac{1}{r} p_{1} \\
=-G_{0} u_{0}^{2}+\eta\left(u_{0}+\frac{1}{r} u_{1}+u_{0} w_{1}\right)-\frac{\eta^{2}}{G_{0}} w_{1} .
\end{gathered}
$$

For $\eta$ sufficiently small, we could seek a series solution in powers of $\eta$. At leading order this yields the solution for a uniformly curved pipe, which is obtained by using the finite Hankel transformation (Mullin \& Greated 1980). In principle this gives an analytic solution, but numerical techniques are needed to invert the transformation. It has not been possible to find an analytical solution for the unsteady case. Alternatively, (4.8)-(4.11) could be solved by expanding in $\alpha^{2}$ and then $\eta$. However, this gives rise to a psuedo-singularity at $O\left(\alpha^{2} \eta\right)$ in the form of a logarithmic term in the pressure which would give rise to an infinite radial pressure gradient at the origin. This implies that a rescaling of the equations at the origin is necessary and then a matching of 
the two regions. However, at present the authors have not been able to resolve this problem. Therefore we have chosen to solve equations (4.8)-(4.11) using numerical techniques.

The numerical method we have chosen is the spectral method, with a basis of Fourier modes. For simplicity we start by changing the variables as follows:

$$
u=r f(\rho, t), \quad v=g(\rho, t), \quad w=h(\rho, t), \quad \text { and } \quad p=r q(\rho, t),
$$

where $\rho=r^{2}$, giving

$$
\begin{gathered}
2 g_{\rho}+\frac{1}{\rho}(g-h)=-\eta f, \\
4 \rho f_{\rho \rho}+8 f_{\rho}-\frac{\alpha^{2}}{2 \pi} f_{t}-2 u_{0, \rho} g=\alpha^{2} \cos 2 \pi t-2 u_{0, \rho}+\eta\left(u_{0}-\frac{\eta}{G_{0}}\right) f+\frac{\eta}{G_{0}} q \\
2 \rho q_{\rho}+q+\frac{\alpha^{2}}{2 \pi} g_{t}+\frac{1}{\rho}(g-h)-2 h_{\rho}=G_{0} u_{0}^{2}-\eta\left(u_{0}+f+2 \rho f\right)-\eta\left(u_{0}-\frac{\eta}{G_{0}}\right) g, \\
4 \rho h_{\rho \rho}+4 h_{\rho}-\frac{\alpha^{2}}{2 \pi} h_{t}+\frac{1}{\rho}(g-h)-2 g_{\rho}-q=-G_{0} u_{0}^{2}+\eta\left(u_{0}+f\right)+\eta\left(u_{0}-\frac{\eta}{G_{0}}\right) h,
\end{gathered}
$$

with the boundary conditions

$$
f=g=h=0 \quad \text { at } \quad \rho=1 \text {; }
$$

we also require all physical quantities to be finite at $\rho=0$.

The above equations are now rearranged into two equations for $f$ and $g$. First we eliminate the pressure terms, $q$, then the $h$ terms in (4.12)-(4.15), giving

$$
\begin{aligned}
\frac{\alpha^{2}}{2 \pi} f_{t} & -\frac{\alpha^{2} \eta^{2} \rho^{2}}{2 \pi G_{0}} f_{t}+\left(\frac{4 \eta^{2} \rho^{2}}{G_{0}}-4 \rho\right) f_{\rho \rho}+\left(\frac{12 \eta^{2} \rho}{G_{0}}-8\right) f_{\rho} \\
- & \eta\left(\left(\frac{\eta^{2} \rho}{G_{0}}-1\right) u_{0}-\frac{\eta}{G_{0}}\left(\frac{\eta^{2} \rho}{G_{0}}+1\right)\right) f-\frac{\alpha^{2} \eta}{2 \pi G_{0}} g_{t}-\frac{\alpha^{2} \eta \rho}{\pi G_{0}} g_{t \rho} \\
+ & \frac{8 \eta \rho^{2}}{G_{0}} g_{\rho \rho \rho}+\frac{28 \eta \rho}{G_{0}} g_{\rho \rho}-\left(\frac{2 \eta^{2} \rho}{G_{0}} u_{0}-\frac{2 \eta^{3} \rho}{G_{0}^{2}}+\frac{8 \eta}{G_{0}}\right) g_{\rho} \\
+ & \left(2 u_{0, \rho}-\frac{\eta^{2}}{G_{0}} u_{0}+\frac{\eta^{3}}{g_{0}^{2}}\right) g-2 u_{0, \rho}+\eta u_{0}^{2}-\frac{\eta^{2}}{G_{0}} u_{0}+\alpha^{2} \cos 2 \pi t=0, \\
\frac{4 \alpha^{2}}{\pi} g_{t \rho} & +\frac{2 \alpha^{2} \rho}{\pi} g_{t \rho \rho}-16 \rho^{2} g_{\rho \rho \rho \rho}-96 \rho g_{\rho \rho \rho}+\left(4 \eta \rho u_{0}-\frac{4 \eta^{2} \rho}{G_{0}}-96\right) g_{\rho \rho} \\
& +\left(4 \eta \rho u_{0, \rho}+8 \eta u_{0}-\frac{8 \eta^{2}}{G_{0}}\right) g_{\rho}+2 \eta u_{0, \rho} g+\frac{3 \alpha^{2} \eta}{2 \pi} f_{t}+\frac{\alpha^{2} \eta \rho}{\pi} f_{t \rho} \\
& -8 \eta \rho^{2} f_{\rho \rho \rho}-44 \eta \rho f_{\rho \rho}+\eta\left(2 \eta \rho u_{0}-2 \frac{\eta^{2} \rho}{G_{0}}-40\right) f_{\rho} \\
& +\eta\left(2 \eta \rho u_{0, \rho}+3 \eta u_{0}-\frac{3 \eta^{2}}{G_{0}}\right) f+4 G_{0} u_{0, \rho} u_{0}+2 \eta u_{0}=0 .
\end{aligned}
$$


We seek solutions which are periodic in time and so we write $f$ and $g$ as Fourier series:

$$
\begin{aligned}
& f(\rho, t)=\frac{a_{f 0}(\rho)}{2}+\sum_{n=1}^{\infty}\left(a_{f n}(\rho) \cos 2 \pi n t+b_{f n}(\rho) \sin 2 \pi n t\right), \\
& g(\rho, t)=\frac{a_{g 0}(\rho)}{2}+\sum_{n=1}^{\infty}\left(a_{g n}(\rho) \cos 2 \pi n t+b_{g n}(\rho) \sin 2 \pi n t\right),
\end{aligned}
$$

where the period is obtained from the forcing terms in (4.16) and (4.17). The spectral approximation is obtained by truncating the summation to $M$ terms. The coefficients of the Fourier series are found by solving the $4(M+1)$ linear ordinary differential equations that are obtained upon taking inner products.

After decomposing the spatial variables using finite difference techniques, the problem reduces to solving

$$
\mathscr{A} \boldsymbol{x}=\boldsymbol{b},
$$

where $\mathscr{A}$ is a block tri-diagonal matrix of coefficients, $\boldsymbol{b}$ is a vector of driving terms and

$$
\boldsymbol{x}=\left[b_{f 0}(\rho), \ldots, b_{f M}(\rho), b_{g 0}(\rho), \ldots, b_{g M}(\rho), a_{f 0}(\rho), \ldots, a_{f M}(\rho), a_{g 0}(\rho), \ldots, a_{g M}(\rho)\right]^{T} .
$$

To extend this model to incorporate the effects of torsion would require another spectral approximation at $O\left(\epsilon^{2}\right)$, which would prove costly in computational terms. Therefore we have not investigated the effects of torsion in the unsteady flow case.

\subsection{Results}

The simplest analytical results to which we can compare the numerical method are those for Hydon's (1994) quasi-steady model. We note that the numerical method agrees well with Hydon's solution for a pipe with uniform curvature. As $\eta$ (the 'nonuniformity' parameter) is decreased, the numerical solution converges to the uniform curvature solution.

First we consider flow in a pipe of uniform curvature $(\eta=0)$ and zero torsion. When $\alpha \ll 1$ the flow is quasi-steady, and therefore the velocity profiles are qualitatively similar to those for steady flow. At $t=0$ the axial component of velocity is at a maximum, the fluid then slows and by $t=0.5$ is completely reversed. The secondary velocity has a two-vortex structure throughout this cycle, but the strength is almost zero at $t=0.25$ (when the unsteady pressure gradient forcing the leading-order flow is zero, i.e. $p_{0 s}=0$ ). The axial component dominates the total wall shear stress (WSS). Maximum axial and total WSS occurs at the outside wall and the minimum WSS occurs at the inside wall. The azimuthal WSS has zeros at these points. When the pressure gradient $\left(p_{0 s}\right)$ is zero there is negligible azimuthal WSS. As the parameter $G_{0}$ is increased the magnitude of the WSS increases linearly at a very slow rate (figure 18). As the Womersley number $\alpha$, is increased the boundary layer/inviscid core structure (first observed by Lyne 1971) forms. This is shown in figure 19.

When $\eta \neq 0$ and $\alpha \ll 1$ the effect of non-uniform curvature is most evident in the secondary velocity and azimuthal wall shear stress (WSS) (figures 20, 21). Due to the non-uniformity of the curvature, the additional driving terms do not all go to zero at $t=0.25$ (unlike the uniform $(\eta=0)$ case). Therefore the secondary velocity is no longer zero at $t=0.25$. The most suprising effect of the non-uniformity is seen at 

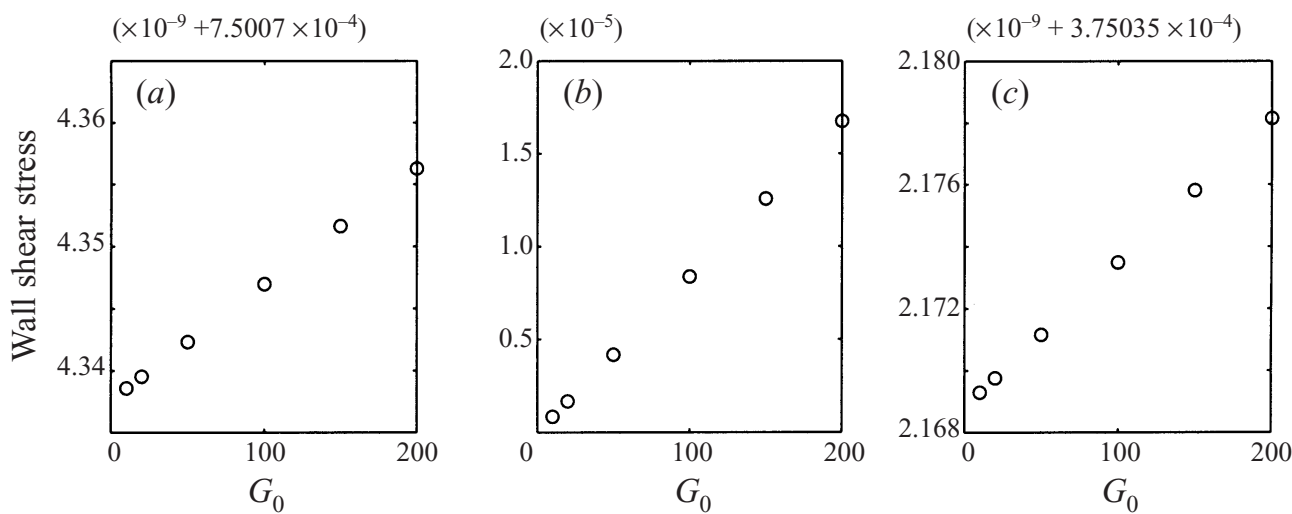

FIGURE 18. Relationship between wall shear stress (WSS) and the parameter $G_{0}$ at $t=0$;

(a) axial; (b) azimuthal; (c) total.
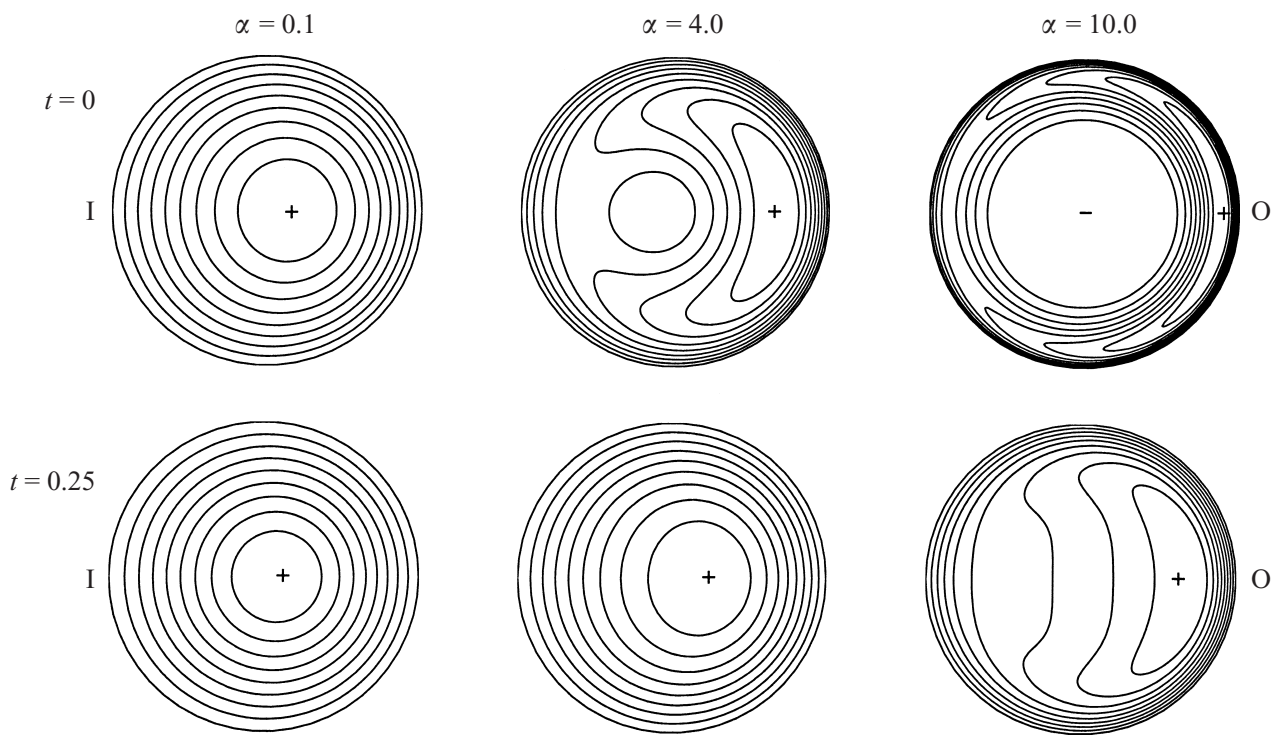

FIGURE 19. Axial velocity contours for a pipe with an unsteady pressure gradient and uniform curvature: $G_{0}=50, \eta=0$.

$t=0.5$ where we observe that the secondary velocity field is reversed and hence the azimuthal WSS is inverted (figure 21). Lyne (1971) showed that a reversal of secondary motion occurs along the centreline when $\alpha$ and $R_{s}$ (the secondary streaming Reynolds number) are large. We find that (in a spiral pipe) when $\alpha$ is large the axial velocity has the boundary layer/inviscid core structure and the secondary flow has a four-vortex structure similar to that described by Lyne (1971). Although the secondary flow is reversed along the centreline, at the wall it is orientated in the same direction as for steady flow. Hence the azimuthal WSS is altered in magnitude but not direction. This is where our results differ significantly from the work of Lyne. When $\alpha \ll 1$ and $\eta \neq 0$, figure 20 shows that, at $t=0.5$, the secondary flow is reversed everywhere in the cross-section; this causes the azimuthal WSS to be inverted (with respect to the $\eta=0$ case) (figure 21).

The reason for this is an inversion of the perturbed pressure profile which can 

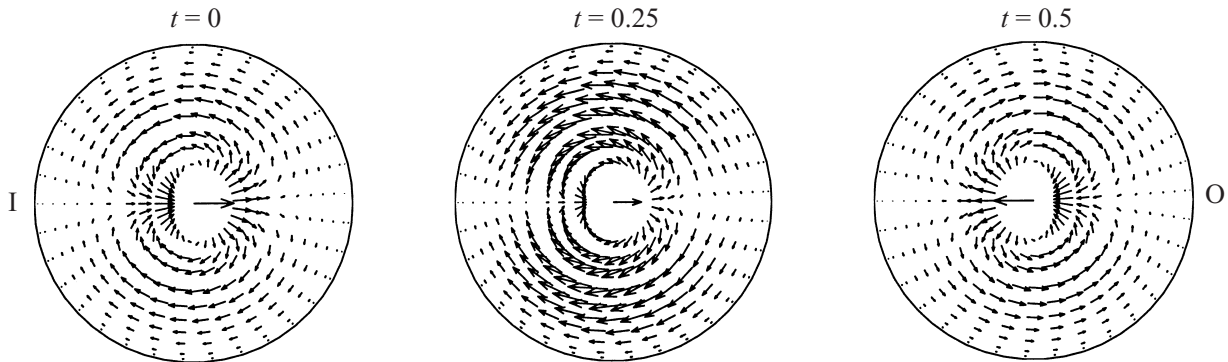

FiguRE 20. Secondary velocity: $\alpha=0.1, G_{0}=150, \eta=0.5$ and $s=5$.
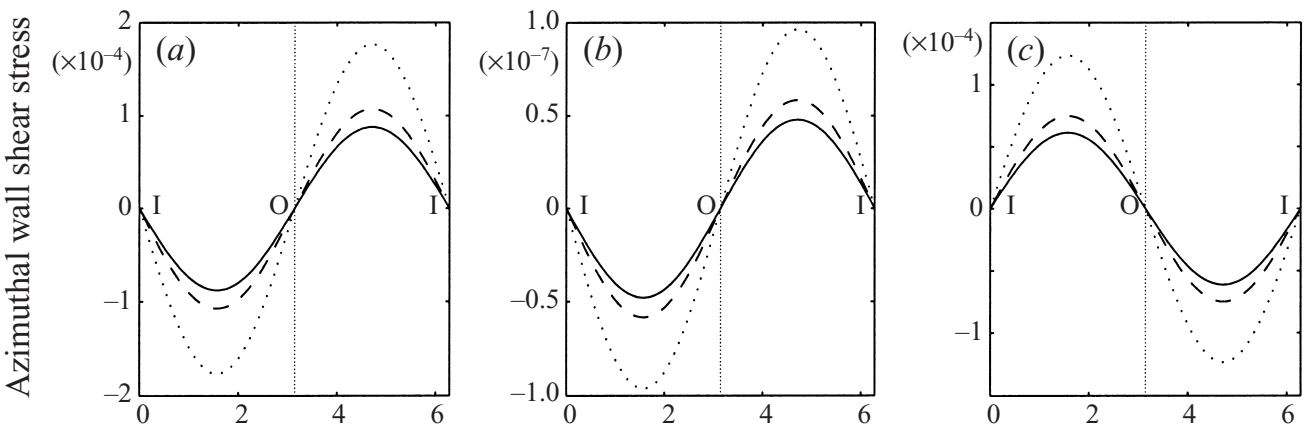

FiguRe 21. Azimuthal wall shear stress. Parameters as for figure 20 with $s=1(-), 5(--), 15(\cdots \cdots)$, and $(a) t=0,(b) t=0.25,(c) t=0.5$.
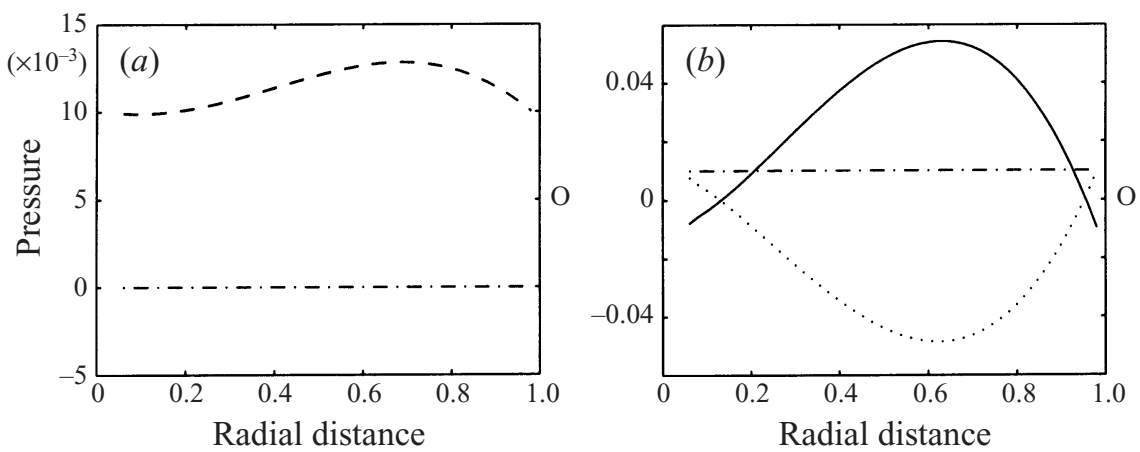

Figure 22. Profiles of pressure, extending from the centre of the cross-section to the outer wall, against radial distance: $(a)$ uniform curvature; $(b)$ non-uniform curvature: $t=0(-), 0.25(\cdot-\cdot-)$, $0.5(\cdots \cdots)$. Note: in $(a)$ the profile is virtually the same at $t=0$ and 0.5 . (Parameters as for figure 20.)

be seen in figure $22(b)$. The pressure profile in a uniformly curved pipe remains approximately the same shape throughout a period (figure $22 a$ ). Therefore, although the strength changes, the direction of the secondary velocity remains unaltered. However, when the pipe has non-uniform curvature the pressure profile is inverted at $t=0.5$ (when reversal is apparent); this causes a change in the sign of the pressure gradient, and hence a change in direction of the secondary flow. As $G_{0}$ is increased, the strength of the secondary flow at $t=0.5$ decreases, and likewise the azimuthal WSS. From figure 23 we see that the amplitude of azimuthal WSS increases (decreases) linearly with $G_{0}$ at $t=0(t=0.5)$. However, the relationship between the axial WSS 

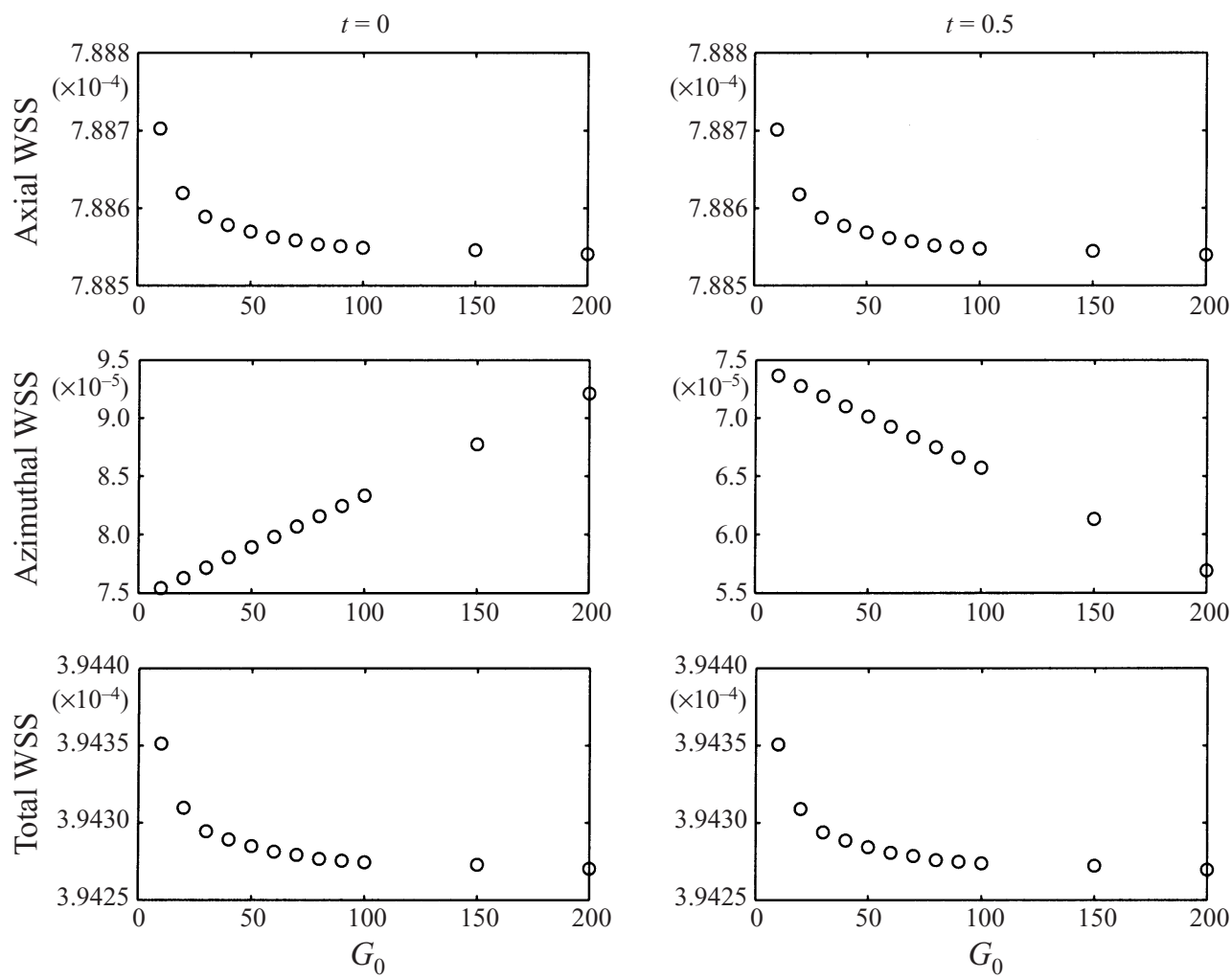

FIGURE 23. The amplitude of WSS vs. $G_{0}$, in a non-uniformly curved pipe: $\alpha=0.1 ; \eta=0.5$.

and $G_{0}$ is no longer linear (figure 23). As $G_{0}$ increases the non-uniformities cease to dominate the flow. As $\alpha$ is increased the non-uniformity has a decreased effect on the flow. When $\alpha \sim 4$ the secondary flow is no longer reversed (at $t=0.5$ ); however both it and the azimuthal WSS are weaker than in pipes with uniform geometry. For $\alpha \geqslant 4$ all shear stresses increase linearly with $G_{0}$. Again, this is due to the dominance of the $\alpha^{2}$ driving terms compared with driving terms occuring due to the non-uniformity. When the boundary layer/core flow structure develops the secondary flow has a greater effect on the WSS. Figure 24 shows that, as the strength of the secondary velocity (and azimuthal WSS) increases, the total WSS has two maxima at $t=0.25$. This effect occurs in both uniform and non-uniform geometries at $\alpha / G_{0} \approx 0.05$; increasing $G_{0}$ or $s$ (in the non-uniform case) pushes the two peaks away from the outer wall. Zabielski \& Mestel (1998a) showed a similar effect in uniform helical pipe flows.

As the arc-length $s$ increases, the magnitudes of the secondary flow and all shear stresses increase; the peak axial velocity is pushed towards the outer wall. This is in agreement with predictions made from the steady-flow case.

\section{Conclusions}

\subsection{Steady flow}

The wall shear stress (WSS) in a weakly curved pipe is dominated by its axial component. When the pipe has weakly non-uniform curvature (with $\left.\epsilon(s)=\kappa_{0} \mathrm{e}^{\eta s}\right)$ the components of WSS increase in magnitude; the azimuthal component is slightly more 

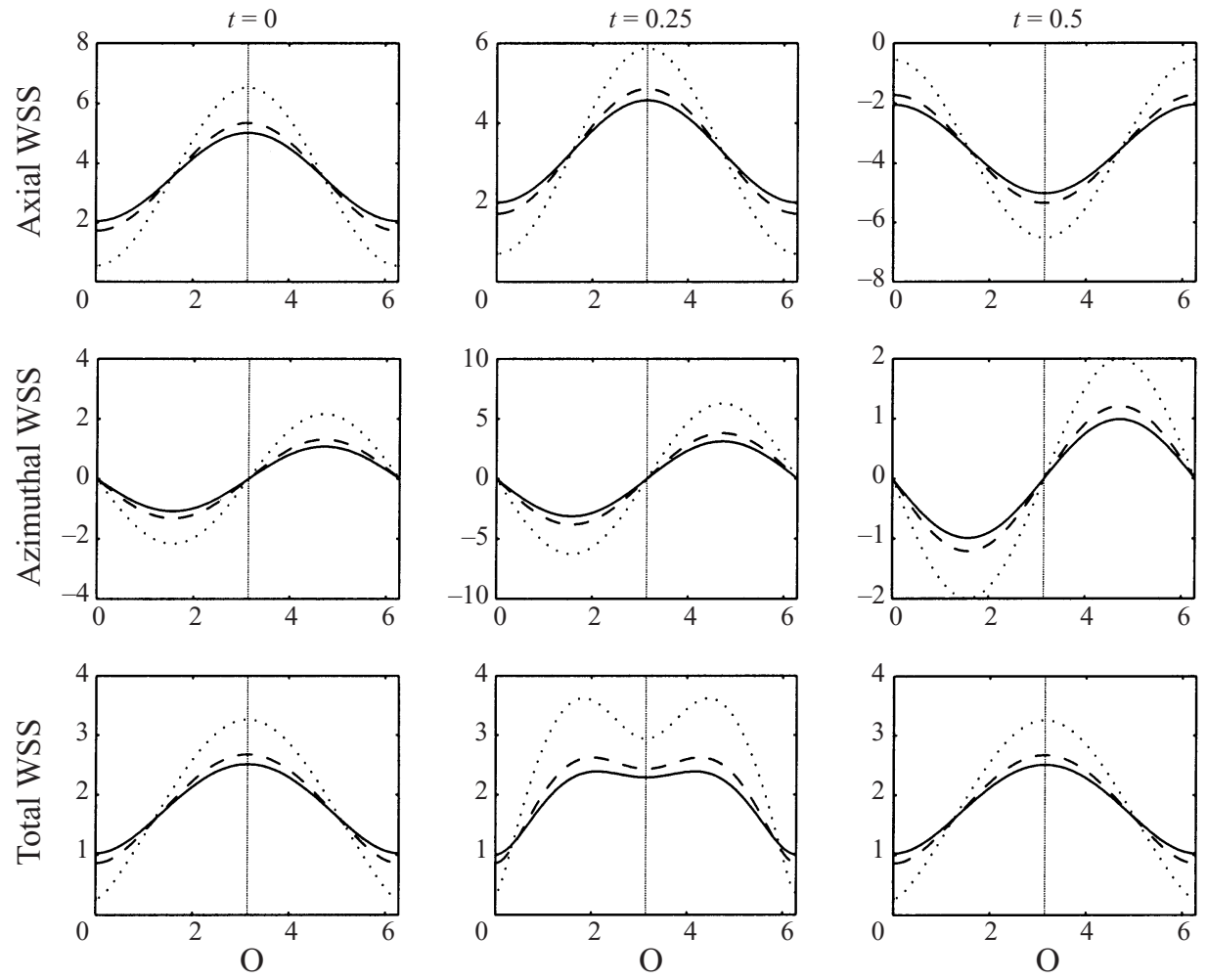

FIGURE 24. Plots of wall shear stress: $\alpha=10 ; G_{0}=150 ; \eta=0$.

affected by the non-uniformity than the axial component. As the arc-length increases, the region of peak axial velocity is forced closer to the outside wall; the secondary velocity increases in magnitude but has unaltered form. When $R e \sim 1$ the viscous driving terms dominate the flow and the region of peak axial velocity is forced closer to the inside wall; the wall shear stress is altered accordingly.

The azimuthal WSS component is dramatically increased by the introduction of torsion. For a uniform pipe, the introduction of torsion breaks the symmetric structure (of flow without torsion) and skews the velocity profiles in the direction of the torsion: the axial component of velocity is also deformed in shape. The azimuthal WSS has an increasing effect on the total WSS as the arc-length $(s)$ increases, causing a 'bump' in the total WSS distribution.

When a pipe has uniform curvature and non-uniform torsion the axial components of velocity and WSS are skewed more than the azimuthal components. The axial component of velocity is not only skewed, but is also increasingly deformed in shape. The azimuthal velocity becomes more skewed in the direction of torsion, but its strength is only slightly increased. The axial component of WSS is skewed more than the azimuthal component and this is highlighted in the altered distribution of the total WSS.

To conclude, we find that as curvature increases, the axial velocity is forced towards the outer wall and the secondary velocity field is increased in strength. Increasing torsion skews the components of velocity in the direction of increasing torsion. 


\subsection{Unsteady flow}

When the pipe has non-uniform curvature and $\alpha<2$, flow reversal occurs. This type of flow reversal is different to that seen by previous authors (e.g. Lyne 1971) because it is due to an inversion of the perturbed pressure profile, whereas Lyne describes reversal along the centreline which is due to the formation of a boundary-layer structure. As $\alpha$ is increased the effect of the non-uniformity weakens and the velocity profiles become approximately the same as for oscillatory flow in a uniformly curved pipe. When $\alpha>4$ the boundary-layer structure forms and when $\alpha / G_{0} \approx 0.05$ the azimuthal WSS has a noticeable effect on the total WSS (at $t=0.25$ ): namely, a double peak is seen in the total WSS (this is true for both uniform and non-uniform pipes).

\subsection{Physiology}

The steady flow calculations have shown that torsion skews and increases the strength of the secondary velocity field, which in turn alters the azimuthal wall shear stress similarly. For oscillating flows it has been shown that torsion raises (from zero) the WSS in certain regions of the pipe (Zabielski \& Mestel 1998b; Gammack 1998). This has a genuine application to bypass graft surgery. It suggests that adding a twist to a graft will reduce the possibility of plaque build-up. The steady flow experiments of C. G. Caro (1998, personal communication) have shown that torsion reduces the size of the downstream stagnation region.

For oscillating flows in non-uniformly curved pipes we have shown that, at low frequencies, the secondary velocity and azimuthal WSS are reversed at a specific time during a period. We have not been able to study large curvature and large frequencies, but we conjecture that a similar reversal would be seen for higher frequencies if the curvature is also sufficiently increased. It would therefore be possible for an in-plane bypass graft to increase the chance of a build-up of atherosclerotic plaques due to the change in direction of WSS (Ku et al. 1985).

\section{Appendix. The $O\left(\epsilon^{2} \lambda\right)$ equations}

The terms of $O\left(\epsilon^{2} \lambda\right)$ in the expansion of Germano's equations are

$$
\begin{aligned}
& \lambda^{-1} \epsilon^{-2}\left(\epsilon^{2} \lambda u_{20}\right)_{s}-u_{1} \cos \xi+v_{20, r}+\frac{w_{20}, \xi}{r}+\frac{v_{20}}{r}=0, \\
& \lambda^{-1} \epsilon^{-2} u_{0}\left(\epsilon^{2} \lambda u_{20}\right)_{s}-u_{0, r} v_{20}-u_{0} u_{1} \cos \xi=-\lambda^{-1} \epsilon^{-2}\left(\epsilon^{2} \lambda p_{20}\right)_{s}+p_{1} \cos \xi \\
& +\frac{1}{\operatorname{Re}}\left[\left(\frac{\partial}{\partial r}+\frac{1}{r}\right)\left[u_{20, r}-\lambda^{-1} \epsilon^{-2}\left(\epsilon^{2} \lambda v_{20}\right)_{s}+v_{1} \cos \xi\right]\right. \\
& \left.+\frac{1}{r} \frac{\partial}{\partial \xi}\left[\frac{u_{20, \xi}}{r}-w_{1} \sin \xi-\lambda^{-1} \epsilon^{-2}\left(\epsilon^{2} \lambda w_{20}\right)_{s}\right]\right], \\
& \lambda^{-1} \epsilon^{-2} u_{0}\left(\epsilon^{2} \lambda v_{20}\right)_{s}-u_{0} v_{1} \cos \xi=-p_{20, r}+\frac{1}{R e}\left[\frac{1}{r}\left[-\frac{w_{20, \xi}}{r}-w_{20, r \xi}+\frac{v_{20, \xi \xi}}{r}\right]\right. \\
& +\lambda^{-1} \epsilon^{-2} \frac{\partial}{\partial s}\left[-\lambda \epsilon^{2} u_{20, r}+\left(\epsilon^{2} \lambda v_{20}\right)_{s}-\lambda \epsilon^{2} v_{1} \cos \xi\right] \\
& \left.-\frac{1}{\epsilon}\left[\left(\epsilon v_{1}\right)_{s} \cos \xi-\epsilon u_{1, r} \cos \xi-\epsilon u_{0} \cos \xi\right]\right],
\end{aligned}
$$




$$
\begin{aligned}
\lambda^{-1} \epsilon^{-2} u_{0}\left(\epsilon^{2} \lambda w_{20}\right)_{s} & +u_{0} w_{1} \sin \xi=-\frac{p_{20, \xi}}{r}+\frac{1}{R e}\left[\frac{w_{20, r}}{r}+w_{20, r r}-\frac{w_{20}}{r^{2}}+\frac{1}{\epsilon}\left(\epsilon w_{1}\right)_{s} \sin \xi\right. \\
& -\frac{u_{1}}{r} \sin \xi-\lambda^{-1} \epsilon^{-2} \frac{\partial}{\partial s}\left(\epsilon^{2} \lambda \frac{u_{20, \xi}}{r}-\epsilon^{2} \lambda w_{1} \sin \xi-\left(\epsilon^{2} \lambda w_{20}\right)_{s}\right) \\
& \left.-\frac{v_{20, r \xi}}{r}+\frac{v_{20, \xi}}{r^{2}}-u_{0} \sin \xi\right] .
\end{aligned}
$$

\section{REFERENCES}

Berger, S. A., Talbot, L. \& Yao, L.-S. 1983 Flow in curved pipes. Ann. Rev. Fluid Mech. 15, 461-512.

BerTelsen, A. F. 1975 An experimental investigation of low Reynolds number secondary streaming effects associated with an oscillating viscous flow in a curved pipe. J. Fluid Mech. 70, 519-527.

Caro, C. G., Doorly, D. J., Tarnawski, M., Scott, K. T., Long, Q. \& Dumoulin, C. L. 1996 Non-planar curvature and branching of arteries and non-planar-type flow. Proc. R. Soc. Lond. A 452, 185-197.

Caro, C. G., Fitz-Gerald, J. M. \& Schroter, R. C. 1971 Atheroma and arterial wall shear: Observation, correlation and proposal of a shear dependent mass transfer mechanism for atherogenesis. Proc. R. Soc. Lond. B 177, 109-159.

Dean, W. R. 1927 Note on the motion of fluid in a curved pipe. Phil. Mag. 4, 208-223.

DEan, W. R. 1928 The stream-line motion of fluid in a curved pipe. Phil. Mag. 5, 673-695.

Dennis, S. C. R. \& NG, M. 1982 Dual solutions for steady laminar flow through a curved tube. Q. J. Mech. Appl. Maths 35, 306-324.

Gammack, D. 1998 Blood flow in twisted arteries. PhD Thesis, University of Surrey, Guildford.

Gerald, C. F. \& Wheatley, P. O. 1994 Applied Numerical Analysis, 5th Edn. Addison Wesley.

Germano, M. 1982 On the effect of torsion on helical pipe flow. J. Fluid Mech. 125, 1-8.

Germano, M. 1989 The Dean equations extended to a helical pipe flow. J. Fluid Mech. 203, 289-305.

Grotberg, J. B. 1984 Volume-cycled oscillatory flow in a tapered channel. J. Fluid Mech. 141, 249-264.

Hüttl, T. J. \& Friedrich, R. $1999 a$ Direct numerical simulation of turbulent flows in curved and helically coiled pipes. In Proc. Third Asian Computational Fluid Dynamics Conference, December 7-11, 1998 Bangalore, India, vol. 2, pp. 183-188.

HütTL, T. J. \& FrIEDRICH, R. 1999b High performance computing of turbulent flow in complex pipe geometries. In High Performance Computing in Science and Engineering '98: Transactions of the High Performance Computing Center Stuttgart (ed. E. Krause \& W. Jäger), pp. 236-251. Springer.

Hüttl, T. J. Wagner, C. \& Friedrich, R. 1999 Navier-Stokes solutions of laminar flows based on orthogonal helical co-ordinates. Intl J. Numer. Meth. Fluids 29, 749-763.

Hydon, P. E. 1994 Resonant advection by oscillatory flow in a curved pipe. Physica D 76, 44-54.

KaO, H. C. 1987 Torsion effect on fully developed flow in a helical pipe. J. Fluid Mech. 184, 335-356.

Ku, D. N., Giddens, D. P., Zarins, C. K. \& Glagov, S. 1985 Pulsatile flow and atherosclerosis in the human carotid bifurcation: Positive correlation between plaque location and low and oscillating shear stress. Arteriosclerosis 5, 293-302.

Larrain, J. \& Bonilla, C. F. 1970 Theoretical analysis of pressure drop in the laminar flow of fluid in a coiled pipe. Trans. Soc. Rheol. 14, 135-147.

LiU, S. \& MASLIYAH, J. H. 1993 Axially invariant laminar flow in helical pipes with finite pitch J. Fluid Mech. 251, 315-353.

Lynch, D. G., Waters, S. L. \& Pedley, T. J. 1996 Flow in a tube with non-uniform, time-dependent curvature: governing equations and simple examples. J. Fluid Mech. 323, 237-265.

Lyne, W. H. 1971 Unsteady viscous flow in a curved pipe. J. Fluid Mech. 45, 13-31.

Mullin, T. \& Greated C. A. 1980 Oscillatory flow in curved pipes. Part 2. The fully developed case. J. Fluid Mech. 98, 397-416.

Murata, S., MiYake, Y. \& InABA, T. 1976 Laminar flow in a curved pipe with varying curvature. J. Fluid Mech. 73, 735-752. 
Nandakumar, K. \& Masliyah, J. H. 1982 Laminar flow through curved tubes. J. Fluid Mech. 119, 475-490.

Pedley, T. J. 1980 The Fluid Mechanics of Large Blood Vessels. Cambridge University Press.

Pedley, T. J. 1995 High Reynolds number flow in tubes of complex geometry with application to wall shear stress in arteries. In Biological Fluid Dynamics, 49th SEB Symposium, pp. 219-241. Society for Experimental Biology.

Schilt, S., Moore Jr, J. E., Delfino, A. \& Meister, J.-J. 1996 The effects of time-varying curvature on velocity profiles in a model of the coronary arteries. J. Biomech. 29, 469-474.

SexL, T. 1930 Über den von E. G. Richarson entdeckten 'Annulareffecckt'. Z. Phys. 61, 349-362.

TutTLE, E. R. 1990 Laminar flow in twisted pipes. J. Fluid Mech. 219, 545-570.

Wang, C. Y. 1981 On the low-Reynolds-number flow in a helical pipe. J. Fluid Mech. 108, 185-194.

WATERS, S. L. 1996 Coronary artery haemodynamics: pulsatile flow in a tube of time-dependent curvature. PhD thesis, University of Leeds, Leeds.

Yamamoto, K., Yanase, S. \& Yoshida, T. 1994 Torsion effect on the flow in a helical pipe. Fluid Dyn. Res. 14, 259-273.

Yanase, S., Gotoh, N. \& Yamamoto, K. 1989 Dual solutions of the flow through a curved tube. Fluid Dyn. Res. 5, 191-201.

Zabielski, L. \& Mestel, A. J. 1998 a Steady flow in a helically symmetric pipe. J. Fluid Mech. 370, 297-320.

ZAbielski, L. \& Mestel, A. J. 1998 b Unsteady blood flow in a helically symmetric pipe. J. Fluid Mech. 370, 321-345.

Zalosh, R. G. \& Nelson, W. G. 1973 Pulsating flow in a curved tube. J. Fluid Mech. 59, 693-705. 